\title{
Coherent scattering and macroscopic coherence: implications for neutrino, dark matter and axion detection
}

\author{
Evgeny Akhmedov, ${ }^{1}$ Giorgio Arcadi, Manfred Lindner and Stefan Vogl \\ Max-Planck-Institut für Kernphysik, \\ Saupfercheckweg 1, 69117 Heidelberg, Germany \\ E-mail: akhmedov@mpi-hd.mpg.de, arcadi@mpi-hd.mpg.de, \\ lindner@mpi-hd.mpg.de, stefan.vogl@mpi-hd.mpg.de
}

ABSTRACT: We study the question of whether coherent neutrino scattering can occur on macroscopic scales, leading to a significant increase of the detection cross section. We concentrate on radiative neutrino scattering on atomic electrons (or on free electrons in a conductor). Such processes can be coherent provided that the net electron recoil momentum, i.e. the momentum transfer from the neutrino minus the momentum of the emitted photon, is sufficiently small. The radiative processes is an attractive possibility as the energy of the emitted photons can be as large as the momentum transfer to the electron system and therefore the problem of detecting extremely low energy recoils can be avoided. The requirement of macroscopic coherence severely constrains the phase space available for the scattered particle and the emitted photon. We show that in the case of the scattering mediated by the usual weak neutral current and charged current interactions this leads to a strong suppression of the elementary cross sections and therefore the requirement of macroscopic coherence results in a reduction rather than an increase of the total detection cross section. However, for the $\nu e$ scattering mediated by neutrino magnetic or electric dipole moments coherence effects can actually increase the detection rates. Effects of macroscopic coherence can also allow detection of neutrinos in $100 \mathrm{eV}$ - a few $\mathrm{keV}$ energy range, which is currently not accessible to the experiment. A similar coherent enhancement mechanism can work for relativistic particles in the dark sector, but not for the conventionally considered non-relativistic dark matter.

Keywords: Neutrino Physics, Beyond Standard Model

ARXIV EPRINT: 1806.10962

\footnotetext{
${ }^{1}$ Also at the National Research Centre Kurchatov Institute, Moscow, Russia.
} 


\section{Contents}

1 Introduction $\quad 1$

1.1 Macroscopic coherence? 2

1.2 Weber's approach and structure factors 4

$\begin{array}{ll}1.3 & \text { Our approach: radiative neutrino scattering on electrons }\end{array}$

$\begin{array}{lll}\text { 1.3.1 Previous studies } & 7\end{array}$

1.3.2 Radiative scattering with $\omega \gtrsim \omega_{\text {char }}$ and phase space constraints $\quad 8$

1.4 The structure of this paper 9

2 Radiative neutrino scattering on electrons $\quad 9$

$\begin{array}{ll}2.1 \text { Weak interactions induced radiative process } & 10\end{array}$

2.2 Radiative scattering and the neutrino magnetic dipole moment 12

3 DM detection through radiative coherent scattering? 14

4 Coherent detection of relativistic axions $\quad 15$

$\begin{array}{llr}5 & \text { Effects of atomic binding } & 16\end{array}$

6 Coherent effects and the cross sections 18

$\begin{array}{lll}7 & \text { Summary and discussion } & 22\end{array}$

$\begin{array}{ll}\text { A Kinematics of the process } & 25\end{array}$

$\begin{array}{ll}\text { B 3-body phase space volume } & 26\end{array}$

$\begin{array}{ll}\text { C Squared matrix elements } & 28\end{array}$

\section{Introduction}

Recently, the COHERENT collaboration has reported the first observation of coherent elastic neutrino-nucleus scattering [1, 2], a process predicted over forty years ago [3, 4]. This observation completed the standard-model picture of neutrino interactions with nucleons and nuclei and opened up a new window to probe physics beyond the standard model and nuclear structure; it also has important implications for astrophysics. Very recently, the CONUS collaboration has reported the first experimental indication of coherent elastic neutrino-nucleus scattering with reactor antineutrinos [5]. Coherence of the process implies that the total cross section is proportional to the squared number of the target particles rather than to their number; as a result, for the first time it became possible to 
observe neutrinos with a hand-held detector rather than with ton- or kiloton-scale ones a spectacular achievement indeed. One then naturally wonders if it is possible to achieve coherence of neutrino detection on scales that are larger than the nuclear scale, such as atomic or even macroscopic scales, leading to a further significant increase of the detection cross sections. This would also be of great interest for detecting Dark Matter (DM) particles which are currently being actively looked for.

Coherent neutrino scattering on atoms [6-8] has a two-fold advantage. First, the scattering would occur not just on nucleons inside the nucleus but also on atomic electrons, and the increased number of scatterers would mean additional enhancement of the detection cross section. Second, within the standard model, $\nu_{e} e$ scattering proceeds through both charged-current $(\mathrm{CC})$ and neutral-current $(\mathrm{NC})$ weak interactions, whereas the $\nu_{\mu, \tau} e$ scattering is mediated only by neutral currents. Therefore, coherent neutrino-atom scattering would be sensitive to neutrino flavour and thus could potentially be used for studying neutrino oscillations. This is in contrast with the already observed coherent elastic neutrino-nucleus scattering proceeding only through neutral-current interactions which are flavour blind.

The problem with coherent neutrino-atom scattering is that the atomic recoil energies would be very small and extremely difficult to measure. Indeed, coherence requires the momentum transfer to the scatterer $|\vec{q}|$ to be smaller than or at most of the order of the inverse radius of the scatterer. It is only under this condition that it will be impossible to find out on which constituent of the target particle has the neutrino scattered, and the neutrino waves scattered from the different constituents will be in phase with each other, which are the necessary conditions for coherent scattering. For neutrino-atom scattering, this would imply

$$
|\vec{q}| \lesssim\left(\text { a few } a_{B}\right)^{-1} \sim 1 \mathrm{keV}
$$

where $a_{B} \simeq 0.53 \AA$ is the Bohr radius. For an atom with the atomic number $A \sim 100$ the recoil energy would then be

$$
E_{\text {rec }} \simeq \frac{\vec{q}^{2}}{2 m_{A}} \sim 10^{-5} \mathrm{eV},
$$

about eight orders of magnitude below the currently achieved sensitivity. Measuring such small recoil energies presents a formidable experimental challenge and, if possible at all, would probably require new technologies.

\subsection{Macroscopic coherence?}

How about scattering with coherence on macroscopic scales? Clearly, this would require measuring even much smaller recoil energies and so does not look practical. It is interesting, however, to inquire what could be the increase of the detection cross sections if such measurements were possible, leaving for the moment the detection problem aside. For an estimate, we will be assuming coherence on the target length scale of $\sim 1 \mathrm{~cm}$ and the target mass $m_{t} \sim 1 \mathrm{~g}$. The total cross section of the elementary neutrino elastic scattering process (i.e. of the scattering on a single target particle) with non-relativistic target particle recoil is $\sigma_{0} \simeq\left(G_{F}^{2} / \pi\right) \omega^{2}$, where $G_{F}$ is the Fermi constant and $\omega$ is the energy of the incident 
neutrino. To achieve macroscopic coherence, we need momentum transfers satisfying $|\vec{q}| \leq$ $q_{0} \sim(1 \mathrm{~cm})^{-1}{ }^{1}$ However, the energies of neutrinos we normally deal with are many orders of magnitude larger than this value, and so are the typical momentum transfers. From the kinematics of elastic scattering it follows that $\vec{q}^{2} \simeq 2 \omega^{2}(1-\cos \theta)$, where $\theta$ is the neutrino scattering angle; therefore, to achieve macroscopic coherence one has to restrict neutrino scattering to nearly forward directions:

$$
1-\cos \theta \leq \frac{\vec{q}_{0}^{2}}{2 \omega^{2}} \ll 1
$$

This means a severe restriction of the phase space accessible to the final-state neutrino, which, in turn, leads to a strong suppression of the corresponding elementary cross section:

$$
\sigma_{0} \simeq \frac{G_{F}^{2}}{\pi} \omega^{2} \longrightarrow \frac{G_{F}^{2}}{2 \pi} q_{0}^{2}
$$

However, in order to find the cross section per one target particle one has to multiply the elementary cross section (1.4) by the number of particles that contribute coherently to the scattering process, i.e. by the number of particles in the coherent volume $L_{0}^{3} \sim 1 / q_{0}^{3}$. As a result, the cross section per target particle will be proportional to $1 / q_{0} \propto N^{1 / 3}$, where $N$ is the number of scatterers in the target and we have assumed that the coherent volume is comparable with the total volume of the target. Thus, by going to smaller $q_{0}$ one could increase the detection cross section. ${ }^{2}$ The total cross section obtained by summing over all the scatterers in the target will then scale as $N^{4 / 3}$, i.e. the cross section increase due to the coherence effects is $\sim N^{1 / 3}$. While this is much smaller than an extra factor of $\sim N$ one could naively expect, it still would mean a very strong enhancement of the detection cross section.

The problem is, of course, that the recoil energies are too small to be detected. For $q_{0} \sim(1 \mathrm{~cm})^{-1} \simeq 2 \times 10^{-5} \mathrm{eV}$ and the total mass of particles in the coherent volume $m_{t} \sim 1 \mathrm{~g}$, one finds $E_{\text {rec }} \sim{\overrightarrow{q_{0}}}^{2} / 2 m_{t} \sim 10^{-43} \mathrm{eV}$, the quantity which is not going to be ever measured. To give just one reason for that, in order to measure recoil energy of this magnitude, one needs an energy resolution of at least the same order of magnitude, $\delta E \sim E_{\text {rec. }}$ By time-energy uncertainty relation, the duration of the measurement process $\delta t$ should then exceed $\sim 10^{27} \mathrm{~s}$, which is about 10 orders of magnitude larger than the age of the Universe. To summarize, macroscopic coherence holds and the cross section becomes very large only for neutrino scattering in a very narrow forward cone, which corresponds to unmeasurably small recoil energies of the target particles.

As is seen from the above discussion, one reason why it is difficult to achieve macroscopic coherence in neutrino scattering processes is that one usually measures the recoil energy of the target particles, which for small recoils is suppressed compared to the recoil

\footnotetext{
${ }^{1}$ Note that by $q_{0}$ we mean the maximum allowed value of $|\vec{q}|$ and not the time component of the 4vector $q$.

${ }^{2}$ Obviously, one cannot go to the limit $q_{0} \rightarrow 0$, as the above estimates are only valid for coherence volumes $1 / q_{0}^{3}$ not exceeding the total volume of the detector.
} 
momentum by a very small factor $\sim q_{0} / 2 m_{t}$. The same applies, of course, to experiments on direct DM detection. ${ }^{3}$

Is it possible to overcome this obstacle by somehow making use of the recoil momentum rather than the recoil energy? In that case no extra suppression factor $q_{0} / 2 m_{t}$ would be there. One such possibility was suggested in the 1980s by Joseph Weber [9-11].

\subsection{Weber's approach and structure factors}

Weber suggested to detect neutrinos through their coherent scattering on crystals in torsion balance experiments. This approach combines two interesting ideas. First, as the force coincides with momentum transfer per unit time, the force neutrinos impinge on a crystal is directly related to the momentum transfer to the target rather than to the recoil energy. As discussed above, this is a very desirable feature. Second, when the expected recoil energy of the individual atoms $E_{\text {rec }}=\vec{q}^{2} / 2 m_{A}$ is below the Debye temperature of the crystal $T_{D}$, the recoil momentum is with high probability given to the crystal as a whole rather than to the individual atoms, similarly to what happens in the Mössbauer effect. Indeed, the recoil-free fraction is approximately given by [12]

$$
f \simeq \exp \left\{-\frac{E_{\mathrm{rec}}}{T_{D}}\left(\frac{3}{2}+\frac{\pi^{2} T^{2}}{T_{D}^{2}}\right)\right\}
$$

where $T$ is the crystal temperature. ${ }^{4}$ For $E_{\text {rec }} \ll T_{D}$ the quantity $f \simeq 1$, i.e. the momentum is transferred to the crystal as a whole with probability close to 1 . For typical Mössbauer crystals $T_{D} \sim 10 \mathrm{keV}$, and the condition $E_{\text {rec }} \ll T_{D}$ is easily satisfied even for neutrinos in the $10 \mathrm{MeV}$ energy range. Weber asserted that, since in this case it is impossible to find out on exactly which atom the neutrino had scattered, the contributions of different scatterers should add up coherently, leading to macroscopic coherence and a very strong enhancement of the detection cross section.

He developed a theoretical approach to describe neutrino coherent scattering on crystals and obtained encouraging results. He then performed experiments with solar neutrinos, reactor antineutrinos and a radioactive neutrino source and in all three cases reported positive results, in reasonable agreement with his theoretical expectations.

These results were met with scepticism, and were strongly criticized by a number of authors. It was pointed out that the same ideas applied to the $X$-ray [13] and neutron [14] scattering on crystals would lead to unrealistically large cross sections in direct contradiction with experiment. In refs. [15-20] the theoretical approach of [9-11] was criticized. It was concluded that the effect had been overestimated by about 24 orders of magnitude. Finally, subsequent torsion balance experiments on neutrino-crystal scattering with sensitivities much higher than the sensitivity of the Weber's device have reported null result $[21,22]$.

\footnotetext{
${ }^{3}$ Macroscopic coherence is, however, readily achieved in experiments on light, $X$-ray or neutron scattering from macroscopic targets because what is detected are the scattered particles and not the recoil of the target.

${ }^{4}$ This formula is valid for harmonic crystals in the limit $T \ll T_{D}$. For a general $T$, the second term in the round brackets in the exponent should be replaced by $6\left(T / T_{D}\right)^{2} \int_{0}^{T_{D} / T} d x x /\left(e^{x}-1\right)$.
} 
So, what went wrong with Weber's ideas? The absence of recoil of the individual atoms, which was the main ingredient of his approach, is necessary for macroscopic coherence, but is not sufficient. It is also necessary that the neutrino waves scattered from different centers be in phase with each other. The amplitudes of particle scattering on a group of scattering centers rather than on a single center should contain the relevant structure factors, which describe the relative phases of the amplitudes corresponding to different scatterers. For elastic neutrino scattering the structure factor is given by

$$
F\left(\vec{k}-\vec{k}^{\prime}\right)=\sum_{i=1}^{N} e^{i\left(\vec{k}-\vec{k}^{\prime}\right) \vec{r}_{i}},
$$

where $\vec{k}$ and $\vec{k}^{\prime}$ are the momenta of the incident and scattered neutrinos, $\vec{r}_{i}$ is the coordinate of the $i$ th scatterer and $N$ is the total number of scatterers in the target. Introducing the number density of scatterers $\rho(\vec{r})=\sum_{i} \delta^{3}\left(\vec{r}-\vec{r}_{i}\right)$, one can rewrite the structure factor (1.6) in the familiar form-factor form

$$
F(\vec{q})=\int d^{3} r e^{i \vec{q} \vec{r}} \rho(\vec{r})
$$

where $\vec{q}=\vec{k}-\vec{k}^{\prime}$ is the momentum transfer to the target.

The structure factors are crucial to the issue of coherence of the scattering process, i.e. to the question of whether the amplitudes of neutrino scattering on different target particles should be added coherently. While the exact form of these factors depend on the specific target utilized in the experiment, the fully coherent and completely incoherent regimes can be studied in a rather general way. Indeed, the squared modulus of the transition amplitude contains the factor

$$
|F(\vec{q})|^{2}=\sum_{i, j=1}^{N} e^{i \vec{q}\left(\vec{r}_{i}-\vec{r}_{j}\right)} .
$$

If the momentum transfer $\vec{q}$ satisfies the condition

$$
\max _{i, j}\left\{\left|\vec{q}\left(\vec{r}_{i}-\vec{r}_{j}\right)\right|\right\} \simeq|\vec{q}| L \ll 1,
$$

(where $L$ is a linear size of the target), one can replace all the phase factors under the sum in eq. (1.8) by unity, which gives $|F(\vec{q})|^{2}=N^{2} .{ }^{5}$ In this case neutrinos scattered from different constituents of the target are in phase with each other. In the opposite limit $|\vec{q}| L \gg 1$ only the diagonal $(i=j)$ terms in the sum survive, and one finds $|F(\vec{q})|^{2} \simeq N$, i.e. we obtain the usual dependence of the total cross section on the number of the target particles. This corresponds to incoherent neutrino scattering.

For scattering on crystals, yet another possibility of having macroscopically coherent effects exists, namely, when the phase differences $\vec{q}\left(\vec{r}_{i}-\vec{r}_{j}\right)$ in eq. (1.8) are integer multiples of $2 \pi$. This leads to the well known Bragg condition for diffraction on crystals,

$$
2 d \sin \vartheta=n \lambda,
$$

\footnotetext{
${ }^{5}$ As discussed above, in the cross section this dependence reduces to $\sim N^{4 / 3}$ if neutrino scattering has to be restricted to nearly forward directions in order to achieve sufficiently small momentum transfers.
} 
where $d$ is the interplanar distance in the crystal, $\vartheta$ is the angle between the neutrino momentum and the atomic plane (the scattering angle being $\theta=2 \vartheta$ ), $\lambda=2 \pi /|\vec{k}|$ and $n$ is an integer. Just like for $X$-ray diffraction on crystals, the intensity of the scattered neutrino wave in the directions of the Bragg maxima is $\propto N^{2}$. It is noticeably different from zero in narrow cones around the Bragg directions, with the corresponding solid angles $\Delta \Omega \propto N^{-2 / 3}$, and is practically zero outside these cones. Thus, the intensity of the scattered neutrino wave around each Bragg maximum is $\propto N^{4 / 3}$ [23]. Since the scattered neutrinos are not detected, the quantity that is in principle measurable is the crystal recoil momentum, or the force impinged on the crystal. For a given direction of the momenta of the incident neutrinos with respect to the crystal atomic planes and $n \neq 0$, eq. (1.10) selects the neutrino energy that satisfies the Bragg condition. ${ }^{6}$ As the Bragg maxima have finite widths, neutrinos in finite energy intervals $\Delta \omega$ will actually experience Bragg diffraction; these intervals are, however, very small and scale as $1 / L \propto N^{-1 / 3}$. As a result, the overall momentum transfer to the crystal scales as $N^{4 / 3} \times N^{-1 / 3}=N$, just like for the scattering on amorphous bodies [23].

Thus, scattering on crystals unfortunately does not give any advantage for neutrino detection, and one is back to consider the condition in eq. (1.9). Since it was not satisfied in Weber's experiments, macroscopic coherence could not be achieved. ${ }^{7,8}$

\subsection{Our approach: radiative neutrino scattering on electrons}

In the present paper we consider a different realization of the idea of employing the momentum transfer to the target rather than the recoil energy of the target particle - radiative neutrino scattering on atomic electrons or on free electrons in a conductor:

$$
\nu+e \rightarrow \nu+e+\gamma .
$$

In this case the emitted photon rather than the recoil electron is detected, and the photon energy $\omega_{\gamma}$ can be as large as the neutrino momentum transfer $\left|\vec{k}-\overrightarrow{k^{\prime}}\right|$. Most importantly, the momentum transfer itself (and so also $\omega_{\gamma}$ ) need not be small in order to ensure macroscopic coherence of the process. What has to be small $\left(\lesssim L_{0}^{-1}\right.$ where $L_{0}$ is the macroscopic length scale of the coherent volume) is the net recoil momentum of the target particle, which is the difference between the momentum transfer from the neutrinos $\vec{k}-\overrightarrow{k^{\prime}}$ and the momentum $\vec{k}_{\gamma}$ carried away by the photon. This can happen even when $\left|\vec{k}-\overrightarrow{k^{\prime}}\right|$ and $\left|\vec{k}_{\gamma}\right|=\omega_{\gamma}$ are both large compared to $L_{0}^{-1}$. The above points directly follow from the expression for the structure factor in the case of the process (1.11) (cf. eq. (1.6)),

$$
F\left(\vec{k}-\vec{k}^{\prime}-\vec{k}_{\gamma}\right)=\sum_{i=1}^{N} e^{i\left(\vec{k}-\vec{k}^{\prime}-\vec{k}_{\gamma}\right) \vec{r}_{i}}
$$

\footnotetext{
${ }^{6}$ For $n=0$ the Bragg condition is satisfied for all neutrino energies. However, it corresponds to forward scattering in which there is no momentum transfer from neutrinos to the crystal.

${ }^{7}$ Note that Weber actually did consider the structure factors, but evaluated them incorrectly [16].

${ }^{8}$ When the preliminary results of this work were presented at CERN neutrino platform week in January 2018, we were informed by P. Huber that some of the considerations presented in sections 1.1 and 1.2 had appeared earlier in the unpublished (but not classified) Jason Report by Callan, Dashen and Treiman [24]. We thank Patrick Huber for this comment and for sending us a scanned copy of [24].
} 
The condition for the scatterers within a volume $\sim L_{0}^{3}$ to contribute coherently is $\mid \vec{k}-\vec{k}^{\prime}-$ $\vec{k}_{\gamma} \mid L_{0} \lesssim 1$.

Note that in both radiative and elastic scattering cases, momentum conservation implies that the argument of the structure factor coincides with the momentum $\vec{p}^{\prime}$ of the recoil electron. This has a simple physical interpretation. As was discussed above, coherence requires $\left|\vec{p}^{\prime}\right| L \ll 1$. Since the uncertainty of the magnitude of momentum cannot be much larger than the momentum itself, we also have in this case $\delta\left|\vec{p}^{\prime}\right| L \ll 1$. The Heisenberg uncertainty relation then means that the coordinate uncertainty of the recoiling electron $\delta x$ exceeds the size of the target, that is, one cannot identify which electron the neutrino was scattered off. The requirement $\left|\vec{p}^{\prime}\right| L \ll 1$ also ensures that the neutrino waves scattered from all the electrons within the volume $L^{3}$ are in phase with each other. These are precisely the conditions of coherence of the contributions of different individual electrons to the amplitude of the process.

\subsubsection{Previous studies}

The radiative neutrino scattering on electrons (1.11) was first considered by Lee and Sirlin back in 1964 [25] and since then has been studied by many authors (see, e.g., [26-32]). To the best of our knowledge, only two studies [28, 29] concern the issue of macroscopic coherence of the process. In [28] it was suggested to use radiative neutrino scattering on free electrons in a conductor in order to detect cosmic background neutrinos. It was argued that macroscopic coherence of the process can be achieved, leading to measurable photon production cross sections. These results have been criticized in [29], where a crucial flaw of [28] was pointed out. It was demonstrated that, as neutrino impact pushes the conduction electrons deeper inside the target, the excess positive ion charge on its surface creates a restoring force which pulls the electrons back. As a result, the cross section of coherent radiative neutrino scattering gets suppressed by a factor $\left(\omega / \omega_{p}\right)^{4}$, where $\omega_{p}=$ $\left(n_{e} e^{2} / m_{e}\right)^{1 / 2} \sim 10 \mathrm{eV}$ is the plasma frequency. For cosmic background neutrinos $\left(\omega / \omega_{p}\right)^{4} \sim$ $10^{-20}$, which makes the process completely unobservable.

It is actually not difficult to understand the reason for this drastic suppression of the photon production cross section. Photon radiation in process (1.11) is due to the time dependent dipole (and in general higher multipole) moments induced by the neutrino scattering on the electrons of the target. In the very long wavelength limit, when the energy transfer to the system (and so also the frequency of the induced radiation) is small compared to the characteristic frequencies of the system, the induced moments are small and the photon radiation is strongly suppressed. This situation is very similar to the one encountered when comparing the cross section of the Rayleigh scattering (photon scattering on bound electrons in atoms) to that of the Thomson scattering (scattering of photons on free electrons). In the classical limit the two cross sections are related by $[33]^{9}$

$$
\sigma_{R} \simeq \frac{\omega^{4}}{\left(\omega_{\mathrm{at}}^{2}-\omega^{2}\right)^{2}} \sigma_{T}
$$

\footnotetext{
${ }^{9}$ The accurate quantum mechanical formula is more complicated and depends sensitively on the atomic structure, see section 5 below. However, the limits of large and small $\omega$ are reproduced by the classical formula (1.13) correctly.
} 
where $\omega_{\text {at }}$ is a characteristic atomic frequency. In the limit $\omega \gg \omega_{\text {at }}$ the two cross sections coincide, i.e. the photon scattering on atomic electrons proceeds as if the electrons were free. In the opposite limit $\omega \ll \omega_{\text {at }}$ one finds $\sigma_{R} \simeq\left(\omega / \omega_{\text {at }}\right)^{4} \sigma_{T} \ll \sigma_{T}$. This is the famous $\omega^{4}$ law which is responsible for the blue color of the sky. The $\sim \omega^{4}$ suppression of the cross section of radiative scattering of cosmic background neutrinos on conduction electrons found in [29] is of exactly the same nature..$^{10}$

\subsubsection{Radiative scattering with $\omega \gtrsim \omega_{\text {char }}$ and phase space constraints}

In the present paper we shall consider neutrino detection through coherent radiative neutrino scattering on atomic electrons or on free electrons in a conductor. We will be assuming the energies of the incident neutrinos to be higher than the corresponding characteristic atomic frequencies $\omega_{\text {at }}$ or plasma frequencies $\omega_{p}$. This will allow the momenta of the emitted photons to exceed $\omega_{\text {at }}$ and $\omega_{p}$, thus avoiding the $\omega^{4}$ suppression of the cross sections discussed above. We will concentrate on the situations when the momentum carried away by the emitted photon nearly compensates the momentum transfer to electrons from neutrinos, leading to very small net recoil momenta of the target electrons. As discussed at the beginning of section 1.3, this will result in macroscopic coherence of the detection process, while completely avoiding the problem of measuring extremely small recoil energies of the target.

There is a price to pay, however. The requirement $\vec{k}-\overrightarrow{k^{\prime}} \simeq \vec{k}_{\gamma}$ puts a stringent constraint on the phase space volume accessible to the final-state particles, and in general also on the amplitude of the process. This should lead to a suppression of the cross section of the individual process, just like in the case of elastic neutrino scattering discussed in section 1.1 (see eq. (1.4)). It has to be seen if the enhancement of the cross section due to macroscopic coherence can overcome this suppression, as it is the case for the elastic neutrino scattering (which, however, is unobservable because of the vanishingly small recoil energies). In the present paper we study this issue in detail.

We find that for radiative neutrino scattering mediated by the standard NC and CC weak interactions macroscopic coherence can occur, but only at the expense of severe restriction of the kinematics of the process, resulting in the net suppression rather than enhancement of the total cross section. In contrast to this, for radiative neutrino scattering mediated by neutrino magnetic or electric dipole moments the net effect is an enhancement of the cross section per target electron compared to that for the elastic scattering, though only for the kinetic energies of electron recoil in the elastic process exceeding $\sim 100 \mathrm{keV}$. In addition, coherent radiative $\nu e$ scattering due to neutrino magnetic or electric dipole moments could potentially allow detection of neutrinos of very low energies, which are currently not accessible to the experiment. The mechanism we consider here is, unfortunately,

\footnotetext{
${ }^{10}$ In ref. [29] this suppression was incorrectly interpreted as being due to the electric neutrality of the target. For neutrino scattering on a charged conductor the restoring force on the electrons accelerated by the neutrino impact would still be there, and would be due to both the pull from the positive ions and push from the excess electrons. As a result, the $\omega^{4}$ suppression would still be present. This is quite analogous to the situation with photon scattering on atomic systems, where the scattering on charged ions exhibits at $\omega \ll \omega_{\text {at }}$ the same $\omega^{4}$ suppression as the scattering on neutral atoms [34, 35].
} 
not operative for conventional (non-relativistic) DM particle candidates; however, it could work for relativistic particles that may exist in the dark sector.

\subsection{The structure of this paper}

The paper is organized as follows. In section 2 we consider the radiative neutrino scattering on free non-relativistic electrons, both without any additional kinematic constraints and assuming that the electron recoil momentum is limited from above by a small value $p_{0}$, allowing for macroscopic coherence of the process. In section 2.1 we discuss the radiative neutrino scattering on free electrons mediated by the usual NC and CC weak interactions, whereas in section 2.2 we study the case when the $\nu e$ scattering is mediated by the neutrino magnetic or electric dipole moments. In section 3 we briefly discuss the question of whether macroscopic coherence could be realized and the same enhancement mechanism could work for direct DM detection and conclude that for conventional DM this is not possible (mainly for kinematic reasons). In section 4 we consider coherent radiative axion-photon conversion due to scattering of relativistic axions on electrons (radiative inverse Primakoff effect). In section 5 we discuss atomic binding effects in the case when radiative scattering takes place on electrons in an atom rather than on free electrons. We demonstrate that these effects can be neglected in the cases of interest to us. In section 6 we use the cross sections obtained in sections 2 and 4 to consider the effects of macroscopic coherence on radiative neutrino scattering and axion-photon conversion processes and the question of whether it can increase the detection cross sections. We summarize and discuss our results in section 7 . Some technical details of our calculations are given in the appendices. The kinematics of radiative $2 \rightarrow 3$ scattering is considered in appendix A, whereas appendix B describes calculations of the integrals over the 3-body phase space. The expressions for the squared matrix elements for the processes studied in the paper are collected in appendix C.

\section{Radiative neutrino scattering on electrons}

We shall consider the process

$$
\nu(k)+e(p) \rightarrow \nu\left(k^{\prime}\right)+e\left(p^{\prime}\right)+\gamma\left(k_{\gamma}\right)
$$

in the rest frame of the initial electron. Here

$$
k=(\omega, \vec{k}), \quad p=(m, \overrightarrow{0}), \quad k^{\prime}=\left(\omega^{\prime}, \vec{k}^{\prime}\right), \quad p^{\prime}=\left(E_{p^{\prime}}, \vec{p}^{\prime}\right), \quad k_{\gamma}=\left(\omega_{\gamma}, \vec{k}_{\gamma}\right)
$$

are the 4-momenta of the incident neutrino, initial-state electron, scattered neutrino, finalstate electron and emitted photon, respectively. In this section we consider radiative neutrino scattering on free non-relativistic electrons; possible effects of atomic binding will be discussed in section 5. Eventually, we will be interested in coherent radiative neutrino scattering on a macroscopic lump of electrons, which we will assume to be unpolarised, i.e. to have zero total spin. This allows us to simplify the problem by neglecting the electron spin, i.e. to consider neutrino scattering on a "spinless electron" - a particle with the electron's charge and mass but zero spin. Neutrinos are assumed to be ultra-relativistic, 
so that the neutrino mass can be neglected both in the kinematics of the process and in calculating transition matrix elements.

We shall consider neutrino-electron scattering mediated either by the usual NC and CC weak interactions or by neutrino magnetic (or electric) dipole moments. In each case we calculate the cross section first allowing all the final-state momenta to span the full ranges allowed by 4 -momentum conservation, ${ }^{11}$ and then restricting the net recoil momentum of the electron to satisfy $\left|\vec{p}^{\prime}\right| \leq p_{0}$, where $p_{0}$ is small compared to the maximum value of $\left|\vec{p}^{\prime}\right|$ allowed by the kinematics of process. We will need the cross sections with such a kinematic restriction when considering macroscopic coherence effects in section 6 .

\subsection{Weak interactions induced radiative process}

In calculating the cross section of radiative neutrino scattering (2.1) on "spinless electron" we take into account only the vector current part of the weak NC and CC interactions of electrons since the axial-vector current does not contribute to neutrino scattering on zerospin targets. The amplitude of the weak interaction induced radiative neutrino scattering on a "spinless electron" can be written as

$$
\mathcal{M}_{w}=-i \frac{G_{F}}{\sqrt{2}} g_{V} e \epsilon_{\mu}^{*}\left(k_{\gamma}\right) Q^{\mu \alpha} j_{\alpha}
$$

Here $g_{V}$ is the vector weak coupling constant, $e$ is the electron charge, $\epsilon^{\mu}\left(k_{\gamma}\right)$ is the polarization vector of the produced photon and

$$
j_{\alpha}=\bar{u}\left(k^{\prime}\right) \gamma_{\alpha}\left(1-\gamma_{5}\right) u(k)
$$

is the matrix element of the neutrino current. To leading order in electroweak interaction the tensor $Q^{\mu \alpha}$ is given by

$$
\begin{aligned}
Q^{\mu \alpha} & =Q^{\mu \alpha}\left(p, k ; p^{\prime}, k^{\prime}, k_{\gamma}\right) \\
& \equiv\left\{\frac{\left(2 p^{\prime}+k_{\gamma}\right)^{\mu}\left(2 p+k-k^{\prime}\right)^{\alpha}}{2 p^{\prime} \cdot k_{\gamma}}-\frac{\left(2 p-k_{\gamma}\right)^{\mu}\left[2 p^{\prime}-\left(k-k^{\prime}\right)\right]^{\alpha}}{2 p \cdot k_{\gamma}}-2 g^{\mu \alpha}\right\} .
\end{aligned}
$$

The subscript $w$ at $\mathcal{M}$ stands for neutrino-electron scattering due to the weak interactions; for $\nu_{\mu} e$ and $\nu_{\tau} e$ scattering the interaction is mediated by the weak neutral current, whereas for the $\nu_{e} e$ scattering both neutral and charged currents contribute. For NC induced radiative $\nu e$ scattering, the leading order amplitude is described by the diagrams of figure 1. The three terms in $Q^{\mu \alpha}$ correspond to the three diagrams shown there. For CC induced radiative scattering of $\nu_{e}$ on a "spinless electron", one cannot directly draw diagrams similar to those in figure 1 , as the vertex $W e \nu$ connecting spin 1,0 and $1 / 2$ fields does not exist. Instead, one should consider the scattering on the "standard" spin $1 / 2$ electron described by the left and middle diagrams of figure 1 with the $Z^{0}$ boson line replaced by the $W^{ \pm}$boson one and the electron and neutrino lines in the final state interchanged. For unpolarised target electrons, in the limit of non-relativistic electron recoils the electron spin becomes

\footnotetext{
${ }^{11}$ Except that for $\omega_{\gamma}$-integrated cross sections an infrared cutoff $\omega_{0}$ will be introduced for the photon energies, see below.
} 


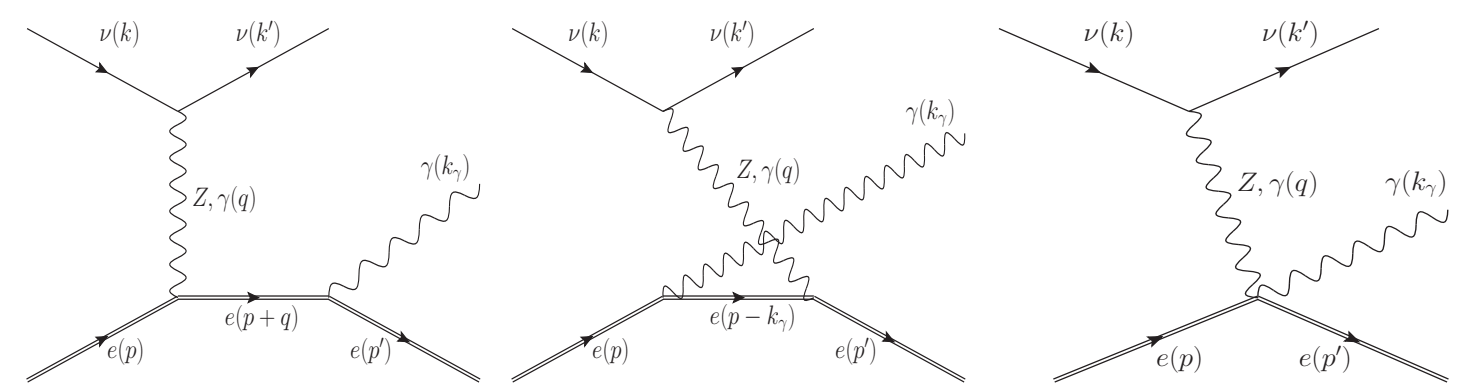

Figure 1. Leading order Feynman diagrams for radiative neutrino scattering (1.11) on a "spinless electron". Shown are the diagrams for $\nu$ e scattering mediated by $Z^{0}$ exchange (NC weak interaction) or photon exchange (for scattering due to the neutrino magnetic or electric dipole moments). The latter case will be considered in section 2.2. For the CC weak interaction contributions, see the text.

relatively unimportant, and the corresponding CC amplitude again has the form (2.3) with $Q^{\mu \alpha}$ given by eq. (2.5). ${ }^{12}$ Note that $Q^{\mu \alpha}$ satisfies the gauge invariance conditions

$$
k_{\gamma \mu} Q^{\mu \alpha}=Q^{\mu \alpha}\left(k-k^{\prime}\right)_{\alpha}=0 .
$$

Thus, expressions (2.3) and (2.5) adequately describe the amplitude of process (1.11) for non-relativistic electrons, with both $\mathrm{NC}$ and $\mathrm{CC}$ contributions properly taken into account. The coupling constant $g_{V}$ is given by

$$
g_{V}=\left\{\begin{array}{l}
2 \sin ^{2} \theta_{W}+\frac{1}{2}, \nu=\nu_{e} \\
2 \sin ^{2} \theta_{W}-\frac{1}{2}, \nu=\nu_{\mu}, \nu_{\tau}
\end{array} .\right.
$$

We now proceed to calculate the cross sections, first without constraining $\left|\vec{p}^{\prime}\right|$. For the double and single differential cross sections one finds

$$
\begin{aligned}
\frac{d^{2} \sigma_{w}}{d \omega_{\gamma} d \cos \theta_{\gamma}} & =\frac{G_{F}^{2} g_{V}^{2} e^{2}}{(2 \pi)^{3}} \frac{1}{m_{e}^{2}} \cdot \frac{1}{3} \frac{\left(\omega-\omega_{\gamma}\right)^{2}}{\omega_{\gamma}}\left\{\left(\omega^{2}-4 \omega \omega_{\gamma}\right)\left(1-\cos ^{2} \theta_{\gamma}\right)+2\left(\omega^{2}+2 \omega_{\gamma}^{2}\right)\right\}, \\
\frac{d \sigma_{w}}{d \omega_{\gamma}} & =\frac{G_{F}^{2} g_{V}^{2} e^{2}}{(2 \pi)^{3}} \frac{1}{m_{e}^{2}} \cdot \frac{8}{9} \frac{\left(\omega-\omega_{\gamma}\right)^{2}}{\omega_{\gamma}}\left\{\left(\omega-\omega_{\gamma}\right)^{2}+\omega^{2}+2 \omega_{\gamma}^{2}\right\} .
\end{aligned}
$$

Here $m_{e}$ is the electron mass and $\theta_{\gamma}$ is the angle between the momentum of the emitted photon and that of the incident neutrino. Because of the usual infrared divergence, in order to calculate the $\omega_{\gamma}$-integrated cross section one has to introduce a lower cutoff for the energy of the emitted photon $\omega_{\gamma \min } \equiv \omega_{0}$. In our case a natural choice of $\omega_{0}$ follows from the requirement that the photon energy exceed the characteristic frequency of the target system, $\omega_{\text {at }}$ for scattering on atomic electrons or $\omega_{p} \sim 10 \mathrm{eV}$ for scattering on free electrons in a conductor. As discussed in section 1.3, this will allow one to avoid the $\sim \omega^{4}$ suppression of the cross section.

\footnotetext{
${ }^{12}$ To arrive at this result one has to make use of the Fierz transformation and consider unpolarised electrons in the limit when their recoil energy is non-relativistic in the rest frame of the initial-state electrons. Note that, as we are interested in coherent effects, the summation over the electron spin states should be done at the amplitude level.
} 
Integration of (2.9) over $\omega_{\gamma}$ yields

$$
\sigma_{w}\left(\omega_{\gamma}>\omega_{0}\right)=\frac{G_{F}^{2} g_{V}^{2} e^{2}}{(2 \pi)^{3}} \frac{1}{m_{e}^{2}} \cdot \frac{16}{9} \omega^{4}\left\{\ln (1 / x)-\frac{41}{24}+3 x-\frac{9}{4} x^{2}+\frac{4}{3} x^{3}-\frac{3}{8} x^{4}\right\},
$$

where

$$
x \equiv \omega_{0} / \omega
$$

For $\omega_{0} \ll \omega$ eq. (2.10) gives

$$
\sigma_{w}\left(\omega_{\gamma}>\omega_{0}\right) \simeq \frac{G_{F}^{2} g_{V}^{2} e^{2}}{(2 \pi)^{3}} \frac{1}{m_{e}^{2}} \cdot \frac{16}{9} \omega^{4}\left[\ln \left(\omega / \omega_{0}\right)-\frac{41}{24}\right] .
$$

Next, we constrain the momentum of the final-state electron by requiring $\left|\vec{p}^{\prime}\right| \leq p_{0}$, where $p_{0}$ is small compared to $\left|\vec{p}^{\prime}\right|_{\max }$ allowed by 4 -momentum conservation. The kinematics of the process in this case is considered in appendix B. As shown there, for a given $\omega_{\gamma}$ the photon emission angle $\theta_{\gamma}$ is now constrained by

$$
0 \leq 1-\cos \theta_{\gamma} \leq \frac{p_{0}^{2}+2 p_{0}\left(\omega-\omega_{\gamma}\right)}{2 \omega \omega_{\gamma}}
$$

The smallness of $p_{0}$ implies that the photons are emitted in nearly forward direction. From the kinematics of the process it follows that the same is true for the scattered neutrino. In the leading order in $p_{0}$ we obtain

$$
\frac{d \sigma_{w}}{d \omega_{\gamma}}=\frac{G_{F}^{2} g_{V}^{2} e^{2}}{(2 \pi)^{3}} \frac{p_{0}^{4}}{4 m_{e}^{2}} \frac{\omega^{2}+\left(\omega-\omega_{\gamma}\right)^{2}}{\omega^{2} \omega_{\gamma}} .
$$

Here the integration over $\cos \theta_{\gamma}$ was performed in its allowed range given in eq. (2.13).

The cross section for the emission of photons with energies $\omega_{\gamma} \geq \omega_{\gamma \min } \equiv \omega_{0}$ reads

$$
\sigma_{w}\left(\omega_{\gamma}>\omega_{0}\right)=\frac{G_{F}^{2} g_{V}^{2} e^{2}}{(2 \pi)^{3}} \frac{p_{0}^{4}}{2 m_{e}^{2}}\left\{\ln (1 / x)-\frac{3}{4}+x-\frac{1}{4} x^{2}\right\} .
$$

For $\omega_{0} \ll \omega$ one can retain only the first two terms in the curly brackets.

The cross sections in eqs. (2.14) and (2.15) scale as the fourth power of $p_{0}$; a factor $p_{0}^{3}$ is expected from the phase space volume of the process with the electron recoil momentum constrained by $\left|\vec{p}^{\prime}\right| \leq p_{0}$ (see appendix B), and one more power of $p_{0}$ comes from the squared modulus of the transition matrix element of the process.

\subsection{Radiative scattering and the neutrino magnetic dipole moment}

Let us now consider the radiative neutrino scattering process (1.11) in the case when the neutrino-electron scattering is mediated by the photon exchange due to neutrino magnetic or electric dipole moments. In what follows we will for definiteness discuss the case of neutrino magnetic dipole moment $\mu_{\nu}$. We will comment on the general case at the end of this subsection. 
The amplitude of the process corresponds to the diagrams in figure 1 in which the intermediate vector boson connecting the neutrino and electron lines is the photon. ${ }^{13} \mathrm{In}$ this case one can expect some kinematic enhancement compared to the usual weak NC and $\mathrm{CC}$ induced processes considered in the previous subsection. Indeed, we are interested in the kinematic region in which the momentum $\vec{k}_{\gamma}$ carried away by the photon nearly coincides with the momentum transfer from the neutrino, $\vec{q}=\vec{k}-\vec{k}^{\prime}$. In the regime of small net recoil momentum of the electron, the same is true for the corresponding energies: $\omega_{\gamma} \simeq \omega-\omega^{\prime}$. This means that the 4-momentum of the virtual photon $q=k-k^{\prime}$ nearly coincides with that of the final-state photon, $k_{\gamma}$. As the produced photon is on the mass shell, the virtual photon is nearly on the mass shell, and its propagator should lead to an enhancement of the amplitude of the process.

The transition matrix element of the neutrino magnetic moment induced radiative scattering process on a "spinless electron" is

$$
\mathcal{M}_{m}=-i e^{2} \frac{\mu_{\nu}}{q^{2}} \epsilon_{\mu}^{*}\left(k_{\gamma}\right) Q^{\mu \alpha} \tilde{j}_{\alpha}, \quad q \equiv k-k^{\prime} .
$$

Here

$$
\tilde{j}_{\alpha}=\bar{u}\left(k^{\prime}\right) \sigma_{\alpha \beta} q^{\beta} u(k)=\left(k+k^{\prime}\right)_{\alpha} \bar{u}\left(k^{\prime}\right) u(k),
$$

where we have used the Gordon identity and took into account that neutrinos are treated as massless particles. As before, the kinematic regime of non-relativistic electron recoil is considered. Without constraining $\left|\vec{p}^{\prime}\right|$, for the double and single differential cross sections we find

$$
\begin{aligned}
\frac{d^{2} \sigma_{m}}{d \omega_{\gamma} d \cos \theta_{\gamma}} & =\frac{\mu_{\nu}^{2} e^{4}}{(2 \pi)^{3}} \frac{1}{4 m_{e}^{2}} \cdot \frac{\left(\omega-\omega_{\gamma}\right)^{2}}{\omega_{\gamma}}\left(3-\cos ^{2} \theta_{\gamma}\right), \\
\frac{d \sigma_{m}}{d \omega_{\gamma}} & =\frac{\mu_{\nu}^{2} e^{4}}{(2 \pi)^{3}} \frac{1}{m_{e}^{2}} \cdot \frac{4}{3} \frac{\left(\omega-\omega_{\gamma}\right)^{2}}{\omega_{\gamma}} .
\end{aligned}
$$

The cross section for the emission of photons with energies $\omega_{\gamma} \geq \omega_{\gamma \min } \equiv \omega_{0}$ is

$$
\sigma_{m}\left(\omega_{\gamma}>\omega_{0}\right)=\frac{\mu_{\nu}^{2} e^{4}}{(2 \pi)^{3}} \frac{1}{m_{e}^{2}} \cdot \frac{4}{3} \omega^{2}\left\{\ln (1 / x)-\frac{3}{2}+2 x-\frac{1}{2} x^{2}\right\}
$$

For $\omega_{0} \ll \omega$ this equation gives

$$
\sigma_{m}\left(\omega_{\gamma}>\omega_{0}\right)=\frac{\mu_{\nu}^{2} e^{4}}{(2 \pi)^{3}} \frac{1}{m_{e}^{2}} \cdot \frac{4}{3} \omega^{2}\left\{\ln \left(\omega / \omega_{0}\right)-\frac{3}{2}\right\}
$$

Next, we again constrain the momentum of the final-state electron by requiring $\left|\vec{p}^{\prime}\right| \leq$ $p_{0}$. Integrating over the $\theta_{\gamma}$ in the allowed range given in eq. (2.13), we find, to leading order in $p_{0}$,

$$
\begin{aligned}
\frac{d \sigma_{m}}{d \omega_{\gamma}} & =\frac{\mu_{\nu}^{2} e^{4}}{(2 \pi)^{3}} \frac{1}{m_{e}^{2}} \cdot \frac{1}{6} \frac{\left(\omega-\omega_{\gamma}\right) p_{0}^{3}}{\omega \omega_{\gamma}^{2}} \\
\sigma_{m}\left(\omega_{\gamma}>\omega_{0}\right) & =\frac{\mu_{\nu}^{2} e^{4}}{(2 \pi)^{3}} \frac{1}{m_{e}^{2}} \cdot \frac{1}{6} \frac{p_{0}^{3}}{\omega_{0}}\{1-x-x \ln (1 / x)\} .
\end{aligned}
$$

\footnotetext{
${ }^{13}$ We ignore the possibility that the final-state photon is emitted from the neutrino line, as this would be a process of higher order in the very small neutrino magnetic moment $\mu_{\nu}$.
} 
For $\omega_{0} \ll \omega$ this gives

$$
\sigma_{m}\left(\omega_{\gamma}>\omega_{0}\right) \simeq \frac{\mu_{\nu}^{2} e^{4}}{(2 \pi)^{3}} \frac{1}{m_{e}^{2}} \cdot \frac{1}{6} \frac{p_{0}^{3}}{\omega_{0}}=\frac{\mu_{\nu}^{2} \alpha^{2}}{\pi} \frac{1}{m_{e}^{2}} \cdot \frac{1}{3} \frac{p_{0}^{3}}{\omega_{0}} .
$$

Interestingly, in this approximation the cross section is essentially independent of the incident neutrino energy $\omega$, except that $\omega_{0}$ should satisfy $\omega_{0}<\omega$.

The cross sections (2.22)-(2.24) increase with decreasing minimum photon energy $\omega_{0}$. Recall, however, that the photon energy cannot be too small: it should exceed the characteristic frequency $\left(\omega_{\mathrm{at}}\right.$ for neutrino scattering and $\omega_{p}$ for scattering on free electrons in a conductor) in order to avoid the $\omega^{4}$ suppression.

As discussed at the beginning of this subsection, the cross section of the neutrino magnetic moment induced process exhibits for small $p_{0}$ a kinematic enhancement due to the propagator of the virtual photon being close to its pole. The enhancement, however, turns out to be relatively mild: the cross sections $(2.22)-(2.24)$ scale as $p_{0}^{3}$, which is to be compared with the $p_{0}^{4}$ dependence found in section 2.1.

We have considered here radiative neutrino scattering process (1.11) induced by the neutrino magnetic dipole moment $\mu_{\nu}$. In general, neutrinos may have both the magnetic and electric dipole moments, which, in addition, are matrices in flavour space. One can take this into account by replacing in the expression for the transition amplitude the quantity $\mu_{\nu}$ by $\tilde{\mu}_{\alpha \beta} \equiv\left(\mu_{\nu}+i \epsilon_{\nu}\right)_{\alpha \beta}$, where $\left(\epsilon_{\nu}\right)_{\alpha \beta}$ is the matrix of neutrino electric dipole moments. Such an amplitude will then describe the transition of $\nu_{\alpha}$ to a neutrino $\nu_{\beta}$ which may be of the same or different flavour. As the final-state neutrino is not detected, in calculating the cross section of the process one has to sum over $\beta$. For the ultra-relativistic neutrinos we confine ourselves to, this amounts to replacing in the expressions for the cross sections $\mu_{\nu}^{2} \rightarrow \sum_{\beta}\left|\tilde{\mu}_{\alpha \beta}\right|^{2}$.

\section{DM detection through radiative coherent scattering?}

It would be interesting to extend the above considerations to detection of other particles, such as DM. Unfortunately, the mechanism of enhancement of the detection cross section through macroscopic coherence considered here for neutrinos would not work for nonrelativistic projectiles. The reason is actually mostly kinematic. Macroscopic coherence requires tiny net recoil momenta $\vec{p}^{\prime}$ of the target electrons. It is easy to see that for non-relativistic projectiles vanishing $\vec{p}^{\prime}$ is excluded by energy-momentum conservation (see appendix A). Small non-zero values of $\vec{p}^{\prime}$ are allowed, but only for extremely soft emitted photons, $\omega_{\gamma} \ll\left|\vec{p}^{\prime}\right|$ (or $\omega_{\gamma} \lesssim\left|\vec{p}^{\prime}\right|$ in the case of moderately relativistic projectiles). As discussed above, the cross sections of radiative scattering on electrons get a very strong $\sim \omega_{\gamma}^{4}$ suppression in this case.

As the conventionally discussed DM particles are supposed to be non-relativistic, the detection enhancement mechanism considered here will not be operative for them. ${ }^{14}$ It may, however, work for detection of relativistic particles that may exist in the dark sector.

\footnotetext{
${ }^{14}$ In ref. [36] it was suggested to use the radiative coherent scattering on nuclei to detect DM particles, but the issue of macroscopic coherence has not been addressed there.
} 

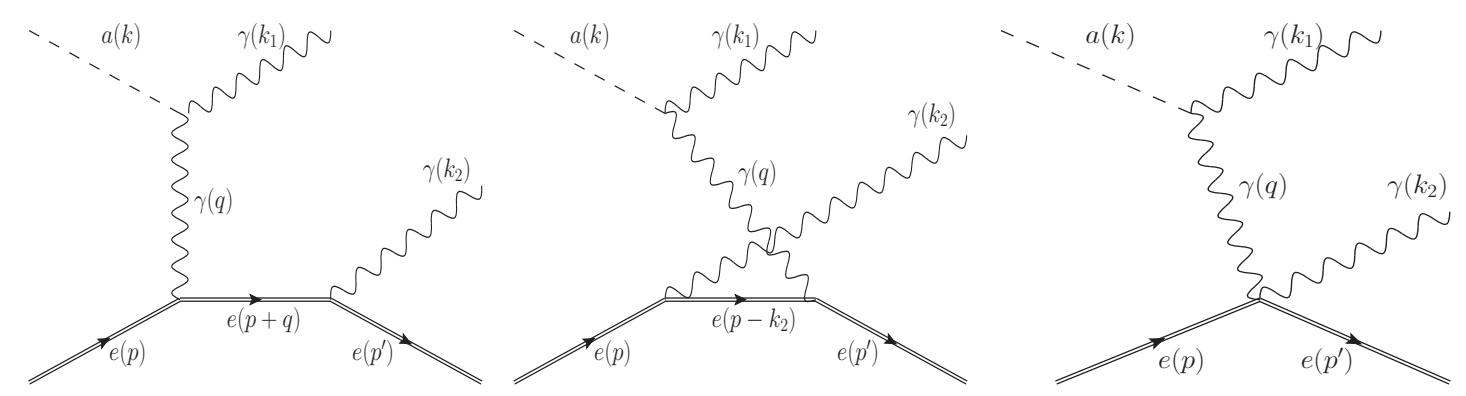

Figure 2. Leading order Feynman diagrams contributing to radiative axion-photon conversion on electrons (4.1) through the inverse Primakoff mechanism. The diagrams with interchanged photon 4-momenta $k_{1}$ and $k_{2}$ should be added.

\section{Coherent detection of relativistic axions}

The enhancement mechanism considered in the present paper could also work for detection of relativistic axions (such as e.g. axions from the sun). The detection process is

$$
a(k)+e(p) \rightarrow e\left(p^{\prime}\right)+\gamma\left(k_{1}\right)+\gamma\left(k_{2}\right) .
$$

In this case there are two photons in the final state, with 4-momenta $k_{1}$ and $k_{2}$.

The mechanism of the process (4.1) that we consider is the radiative inverse Primakoff effect. As before, we will be interested in the coherent interaction of the projectile particle with a group of electrons of zero total spin, which allows us to adopt the approximation of a "spinless electron". The leading order diagrams contributing to the process are shown in figure 2. Note that the non-radiative inverse Primakoff effect on charged particles is incoherent, as the contributions of scattering on electrons and positively charged nuclei cancel each other in the case of electrically neutral targets for low momentum transfers [37]. Coherent axion-photon conversion is, however, possible in external magnetic fields [38]. We do not consider Compton-like photon production (or double photon production) because the direct axion-electron coupling is spin dependent and so it cannot be coherently enhanced in the case of unpolarized targets.

We consider the $a \gamma \gamma$ interaction Lagrangian

$$
\mathcal{L}=\frac{1}{4} g_{a \gamma \gamma} a F_{\mu \nu} \tilde{F}^{\mu \nu}
$$

The amplitude of the process corresponding to the diagrams of figure 2 can be written in the form similar to (2.16):

$$
\mathcal{M}_{a}=-i e^{2} g_{a \gamma \gamma}\left\{\frac{1}{q_{1}^{2}} \epsilon_{\mu}^{*}\left(k_{1}\right) Q^{\mu \alpha}\left(p, k ; p^{\prime}, k_{1}, k_{2}\right) \hat{j}_{2, \alpha}+\frac{1}{q_{2}^{2}} \epsilon_{\mu}^{*}\left(k_{2}\right) Q^{\mu \alpha}\left(p, k ; p^{\prime}, k_{2}, k_{1}\right) \hat{j}_{1, \alpha}\right\} .
$$

Here

$$
\hat{j}_{i, \alpha}=\varepsilon_{\rho \beta \sigma \alpha} k_{i}^{\rho} \epsilon^{* \beta}\left(k_{i}\right) q^{\sigma}, \quad q_{i} \equiv k-k_{i},
$$

and the tensor $Q^{\mu \alpha}\left(p, k ; p^{\prime}, k_{1}, k_{2}\right)$ was defined in eq. (2.5). We will neglect the axion mass $m_{a}$ in all the calculations, except that we will keep the $g_{a \gamma \gamma}$ coupling constant (which is 
usually assumed to be proportional to $m_{a}$ ) finite. As before, we calculate all the cross sections in the regime of non-relativistic electrons, first without any additional constraints on $\left|\vec{p}^{\prime}\right|$. For detection of one of the two emitted photons the double and single differential cross section are

$$
\begin{aligned}
\frac{d^{2} \sigma_{a}}{d \omega_{1} d \cos \theta_{1}}= & \frac{g_{a \gamma \gamma}^{2} e^{4}}{(2 \pi)^{3}} \frac{1}{96 m_{e}^{2} \omega \omega_{1}\left(\omega-\omega_{1}\right)}\left\{4 \omega^{4}-16 \omega^{3} \omega_{1}+25 \omega^{2} \omega_{1}^{2}-17 \omega \omega_{1}^{3}+8 \omega_{1}^{4}\right. \\
& \left.+\omega \omega_{1}\left[\left(3 \omega^{2}-9 \omega \omega_{1}+10 \omega_{1}^{2}\right) \cos \theta_{1}+3\left(\omega-\omega_{1}\right)^{2} \cos ^{2} \theta_{1}\right]\right\} \\
\frac{d \sigma_{a}}{d \omega_{1}}= & \frac{g_{a \gamma \gamma}^{2} e^{4}}{(2 \pi)^{3}} \frac{1}{12 m_{e}^{2} \omega \omega_{1}\left(\omega-\omega_{1}\right)}\left(\omega^{4}-\frac{15}{4} \omega^{3} \omega_{1}+\frac{23}{4} \omega^{2} \omega_{1}^{2}-4 \omega \omega_{1}^{3}+2 \omega_{1}^{4}\right) .
\end{aligned}
$$

To find the total cross section of the process we have to integrate (4.6) over $\omega_{1}$ in the interval $\left[\omega_{0}, \omega-\omega_{0}\right]$. The upper integration limit is $\omega-\omega_{0}$ rather than $\omega$ because we now have two photons in the final state, whose energies are related by the energy conservation condition $\omega_{1}+\omega_{2}=\omega$ and must both be above the infrared cutoff $\omega_{0}$. For the total cross section we then find

$$
\sigma_{a}=\frac{e^{4} g_{a \gamma \gamma}^{2}}{(2 \pi)^{3}} \frac{\omega^{2}}{6 m_{e}^{2}}\left\{\ln \left(\frac{1}{x}-1\right)-\frac{41}{24}+\frac{15}{4} x-x^{2}+\frac{2}{3} x^{3}\right\}
$$

with $x=\omega_{0} / \omega$. Note that $x$ must satisfy $x<1 / 2$ as otherwise energy conservation would force one of the photon energies to be below $\omega_{0}$ in violation of our assumption.

Next, we consider the case in which $\left|\vec{p}^{\prime}\right|$ is constrained from above by a small value $p_{0}$. For the differential and total cross sections we find

$$
\frac{d \sigma_{a}}{d \omega_{1}}=\frac{g_{a \gamma \gamma}^{2} e^{4}}{(2 \pi)^{3}} \frac{p_{0}^{3}}{48 m_{e}^{2} \omega^{2}}\left(\frac{\omega_{1}}{\omega-\omega_{1}}+\frac{\omega-\omega_{1}}{\omega_{1}}\right)
$$

and

$$
\sigma_{a}=\frac{g_{a \gamma \gamma}^{2} e^{4}}{(2 \pi)^{3}} \frac{p_{0}^{3}}{24 m_{e}^{2} \omega}\left[\ln \left(\frac{1}{x}-1\right)-1+2 x\right]
$$

\section{$5 \quad$ Effects of atomic binding}

In sections 2 and 4 we considered radiative neutrino scattering and radiative axion-photon conversion on free electrons. This is suitable for conduction electrons in metals; however, for scattering on atomic electrons in dielectrics the effects of atomic binding should in general be taken into account. We shall show now that for the kinematic regime of interest to us, when the net recoil momentum of the electron is small and at same time the neutrino or axion energy satisfies $\omega \gg \omega_{\text {at }}$, the atomic effects can be neglected and the results found in section 2 apply.

To demonstrate this, let us first note that for radiative scattering on free electrons, in the regime of small $\left|\vec{p}^{\prime}\right|$ the contribution of the first two terms in the expression for $Q^{\mu \alpha}(2.5)$ is small, and the main contribution comes from the third term, corresponding 
to the right diagram of figure $1 .^{15}$ The same holds true when atomic effects are taken into account: in the kinematic region of interest to us the analogues of the first two terms in $Q^{\mu \alpha}$ are small, and the main contribution comes form the analogue of the third term, which is largely insensitive to the effects of atomic structure (see below). This is fully analogous to what happens for elastic scattering of photons on atoms when the photon energy is much higher than the characteristic atomic frequencies $\omega_{\text {at. }}$. As discussed in section 1.3, in this limit the cross section essentially coincides with that of photon scattering on free electrons. This can be readily seen from the expression for the amplitude of elastic photonatom scattering. For non-relativistic electrons, the leading order amplitude in the Coulomb gauge is proportional to [39, 40]

$$
\begin{aligned}
& -\frac{1}{m} \sum_{n}\left\{\frac{\left\langle i\left|e^{-i \vec{k}_{f} \vec{r}} \overrightarrow{\mathrm{p}}_{f}^{*}\right| n\right\rangle\left\langle n\left|e^{i \vec{k}_{i} \vec{r}} \overrightarrow{\mathrm{p}} \vec{\epsilon}_{i}\right| i\right\rangle}{E_{n}-E_{i}-\omega_{i}-i \varepsilon}+\frac{\left\langle i\left|e^{i \vec{k}_{i} \vec{r}} \overrightarrow{\mathrm{p}} \vec{\epsilon}_{i}\right| n\right\rangle\left\langle n\left|e^{-i \vec{k}_{f} \vec{r}} \overrightarrow{\mathrm{e}}_{f}^{*}\right| i\right\rangle}{E_{n}-E_{i}+\omega_{i}-i \varepsilon}\right\} \\
& +\left(\vec{\epsilon}_{f}^{*} \cdot \vec{\epsilon}_{i}\right)\left\langle i\left|e^{i\left(\vec{k}_{i}-\vec{k}_{f}\right) \vec{r}}\right| i\right\rangle .
\end{aligned}
$$

Here $\overrightarrow{\mathrm{p}}=-i \vec{\nabla}$ is the 3 -momentum operator, $\vec{k}_{i}$ and $\vec{k}_{f}$ are the momenta of the incident and scattered photons, $\vec{\epsilon}_{i}$ and $\vec{\epsilon}_{f}$ are their polarizations vectors, and the sum is over the intermediate atomic states. In eq. (5.1) we have taken into account that for elastic scattering on a heavy system $\omega_{f}=\left|\vec{k}_{f}\right|$ coincides with $\omega_{i}=\left|\vec{k}_{i}\right|$. For $\omega_{i} \ll \omega_{\text {at }}$ all three terms in (5.1) are of the same order of magnitude and nearly cancel each other, leading to the $\sim \omega_{i}^{4}$ suppression mentioned in section 1.3; however, in the regime $\omega_{i} \gg \omega_{\text {at }}$ that is of interest to us, the first two terms in (5.1) are small compared to the third term and to a good accuracy can be neglected. Moreover, for spherically symmetric atomic states $|i\rangle$ they tend to cancel each other. ${ }^{16}$ The remaining term, $\left(\vec{\epsilon}_{f}^{*} \cdot \vec{\epsilon}_{i}\right)\left\langle i\left|e^{i\left(\vec{k}_{i}-\vec{k}_{f}\right)} \vec{r}\right| i\right\rangle$, in general depends on the electron charge distribution in the state $|i\rangle$. For $\left|\vec{k}_{i}-\vec{k}_{f}\right| \ll R_{\mathrm{at}}^{-1}$ it is actually independent of the atomic structure, and for photon scattering on a single atom reduces to $Z\left(\vec{\epsilon}_{f}^{*} \cdot \vec{\epsilon}_{i}\right)$, where $Z$ is the total number of the atomic electrons. This corresponds to coherent elastic photon-atom scattering. If the more stringent condition $\left|\vec{k}_{i}-\vec{k}_{f}\right| \ll L^{-1}$ is satisfied where $L$ is the linear size of the target, the scattering on all electrons in the target is coherent. Otherwise, one would need to take into account structure factors describing electron distribution in the target, as discussed in section 1.2.

\footnotetext{
${ }^{15}$ Indeed, for small electron recoil momenta the terms in $Q^{\mu \alpha}$ proportional to $p^{\prime \mu} p^{\alpha}$ and to $p^{\mu} p^{\prime \alpha}$ nearly cancel each other, whereas the terms $\propto\left(k-k^{\prime}\right)^{\alpha}$ are subleading in the case of non-relativistic electrons and weak NC and CC mediated neutrino-electron scattering (their contributions vanish exactly for neutrino magnetic moment mediated $\nu e$ scattering as well as for axion-photon conversion). The terms $\propto k_{\gamma}^{\mu}$ do not contribute by gauge invariance. Note that for calculations in the Coulomb gauge in the rest frame of the initial-state electron the whole second term in $Q^{\mu \nu}$ does not contribute to the amplitude, and the contribution of the first term is small because of $p^{\prime} \approx p$.

${ }^{16}$ Indeed, using the closure property of the atomic states and commuting the factors $e^{i \vec{k}_{i} \vec{r}}, e^{-i \vec{k}_{f} \vec{r}}$ with the momentum operator, for the sum of the first two terms in (5.1) one finds in this limit $-\left(1 / m \omega_{i}\right) \epsilon_{f}^{* l} \epsilon_{i}^{s} e^{i\left(\vec{k}_{i}-\vec{k}_{f}\right) \vec{r}}\left\langle i\left|k_{i}^{l} \mathrm{p}^{s}+k_{f}^{s} \mathrm{p}^{l}\right| i\right\rangle$, which vanishes for spherically symmetric states $|i\rangle$. As we are interested in coherent scattering on a group of atoms, by $|i\rangle$ one should actually understand the ground state of such a system. The cancellation then happens also in the case when this state is spherically symmetric (i.e. has zero total angular momentum), even if the ground states of the individual atoms are not.
} 
Similar arguments apply to radiative neutrino scattering on atoms. Note that in this case one has to replace in eq. (5.1)

$$
\vec{k}_{i} \rightarrow \vec{k}-\vec{k}^{\prime}, \quad \omega_{i} \rightarrow \omega-\omega^{\prime}, \quad \vec{k}_{f} \rightarrow \vec{k}_{\gamma}, \quad \omega_{f} \rightarrow \omega_{\gamma}, \quad \vec{\epsilon}_{i} \rightarrow \vec{j} \quad \text { and } \quad \overrightarrow{\mathrm{p}} \vec{\epsilon}_{i} \rightarrow \mathrm{p} \cdot \mathrm{j},
$$

where $\mathrm{p}^{\mu}=i \partial^{\mu}$ is the 4-momentum operator and $j^{\mu}=\left(j^{0}, \vec{j}\right)$ is the relevant matrix element of the neutrino current. The condition $\left|\vec{k}_{i}-\vec{k}_{f}\right| \ll R_{\mathrm{at}}^{-1}$ is then replaced by $\left|\vec{k}-\vec{k}^{\prime}-\vec{k}_{\gamma}\right|=\left|\vec{p}^{\prime}\right| \ll R_{\text {at }}^{-1}$, which we always assume to be satisfied with a large margin when discussing macroscopically coherent effects. With minor modifications related to the presence of two photons in the final state, the same argument applies also to radiative axion-photon conversion on atoms.

It should be noted that for $\omega \gg \omega_{\text {at }}$ inelastic scattering with ionization or excitation of atoms typically dominates, while the processes in which the atom remains in its initial state are only important for nearly forward scattering. This is, however, exactly the case we are interested in. The fact that the probability of the radiative scattering without excitation or ionization of the target atoms is small is already taken into account by the suppression of the individual cross sections which we found upon constraining the electron recoil momentum by $\left|\vec{p}^{\prime}\right| \lesssim p_{0} \sim 10^{-5} \mathrm{eV}$.

\section{Coherent effects and the cross sections}

Let us now assess the effects of macroscopic coherence on the cross sections of neutrino and axion detection processes (2.1) and (4.1).

As discussed in sections 1.2 and 1.3, in order to take possible macroscopic coherence effects into account one has to multiply the elementary amplitude of the process by the relevant structure factor (such as (1.6) or (1.12)). The structure factor depends on the target used in the experiment, and the calculated cross section will therefore also be targetdependent. However, simple estimates of the effects of macroscopic coherence can be obtained in a rather general way as follows.

Assume that all the scatterers contained in some volume of a linear size $L_{0}$ within the target contribute to the cross section coherently; for this to occur, the net recoil momentum of the scatterer $\vec{p}^{\prime}$ must satisfy $\left|\vec{p}^{\prime}\right| \lesssim p_{0} \sim 2 \pi L_{0}^{-1}$. The coherent volume $L_{0}^{3}$ can in principle range from just the volume per one scatterer (no coherence) to the total volume of the target $L^{3}$ (complete coherence). To assess the coherence effects one can first calculate the elementary cross section of the process with the constraint $\left|\vec{p}^{\prime}\right| \leq p_{0}$ imposed. In calculating such constrained elementary cross sections the corresponding structure factors can be replaced by unity. To find the cross section per target particle with coherence effects taken into account one would then have to multiply the constrained elementary cross section by the number of scatterers in the coherent volume $L_{0}^{3} \simeq\left(2 \pi / p_{0}\right)^{3}{ }^{17}$ The

\footnotetext{
${ }^{17}$ Let the number of scatterers within one coherent volume be $N_{0}$, and the number of coherent volumes in the target be $k$. The total number of scatterers in the target is $N=k N_{0}$. If $\sigma_{0}$ is the elementary cross section of the process, the cross section corresponding to scattering on all the target particles contained within one coherent volume is $\sigma_{0} N_{0}^{2}$. The total cross section is $\sigma_{0} N_{0}^{2} \times k=\sigma_{0} N_{0} N$. The cross section per one target particle is then $\sigma_{0} N_{0}$, as stated. In the fully coherent case $\left(N_{0}=N\right)$ and completely incoherent case $\left(N_{0}=1\right)$ the total cross sections are $\sigma_{0} N^{2}$ and $\sigma_{0} N$, respectively, and the corresponding cross sections per target particle are $\sigma_{0} N$ and $\sigma_{0}$.
} 
choice of the recoil momentum cutoff $p_{0}$ (i.e. of the linear size of $L_{0}$ of the coherent volume) would then have to be optimized, within the range allowed by the kinematics of the process and the geometry of the experiment, by maximizing the resulting cross section.

In doing this, one should not forget the issue of observability of the process, which may be fully coherent but completely unobservable. For example, as discussed in section 1.1, for elastic neutrino scattering the optimization requires to choose for the maximum recoil momentum (denoted $q_{0}$ there) the smallest possible value $q_{0} \sim L^{-1}$, but the scattering will then be unobservable due to the vanishingly small recoil energy of the target particles (see the discussion around eqs. (1.3) and (1.4)). No such problems arise for radiative processes discussed in the present paper.

We shall now estimate the effects of possible macroscopic coherence on radiative neutrino scattering on electrons. The corresponding cross sections with the net electron recoil momentum constrained by $\left|\vec{p}^{\prime}\right| \leq p_{0}$ with a small cutoff $p_{0}$ were found in section 2 . Consider first radiative neutrino-electron scattering mediated by the usual NC and CC weak interactions. The constrained differential and integrated elementary cross sections are given in eqs. (2.14) and (2.15), and are proportional to $p_{0}^{4}$. To find the cross section per one target electron one has to multipy these cross sections by the number of electrons in the coherent volume,

$$
N_{0 e} \simeq n_{e} L_{0}^{3} \simeq n_{e}\left(\frac{2 \pi}{p_{0}}\right)^{3},
$$

where $n_{e}$ is the electron number density in the target. As a result, the cross sections per one target electron turn out to be proportional to $p_{0}$ and are maximized for maximal possible value of $p_{0}$, which corresponds to the absence of macrosopic coherence. What actually happens in this case is that macroscopic coherence can be achieved, but it requires such a stringent constraint on the value of $\left|\vec{p}^{\prime}\right|$ (and so on the phase space available to the final-state particles) that the resulting cross sections are much smaller than those in the incoherent case. That is, macroscopic coherence is possible, but it leads to a reduction of the cross section rather than to its increase.

The situation is different for neutrino magnetic (or electric) dipole moment mediated radiative neutrino scattering. As discussed in section 2.2 , for small $\vec{p}^{\prime}$ the cross sections get an enhancement due to the propagator of the virtual photon being close to its massshell pole. The enhacement is, however, rather modest: the constrained elementary cross sections $(2.22)-(2.24)$ are proportional to $p_{0}^{3}$ rather than to $p_{0}^{4}$, as it was in the case of weak $\mathrm{NC}$ and $\mathrm{CC}$ mediated radiative process. As before, to obtain the cross sections per target electron we have to multiply the constrained elementary cross sections by $N_{0 e}$ given by eq. (6.1). The factor $p_{0}^{3}$ in the cross sections $(2.22)-(2.24)$ then gets canceled by $1 / p_{0}^{3}$ from eq. (6.1), i.e. to leading order in the small $p_{0}$ the resulting cross sections per target electron are $p_{0}$-independent. ${ }^{18}$ From eqs. (2.22) and (2.24) we then find

$$
\frac{d \bar{\sigma}_{m}}{d \omega_{\gamma}} \simeq \frac{\mu_{\nu}^{2} e^{4}}{6} \frac{\left(\omega-\omega_{\gamma}\right)}{\omega \omega_{\gamma}^{2}} \frac{n_{e}}{m_{e}^{2}},
$$

\footnotetext{
${ }^{18}$ As follows from the derivation of eqs. $(2.22)-(2.24)$, this is correct only when $p_{0}$ satisfies $L^{-1} \lesssim p_{0} \ll$ $\omega, \omega_{\gamma}, \omega-\omega_{\gamma}$.
} 


$$
\bar{\sigma}_{m}\left(\omega_{\gamma}>\omega_{0}\right) \simeq \frac{1}{6} \mu_{\nu}^{2} e^{4} \frac{n_{e}}{m_{e}^{2} \omega_{0}}=\frac{8}{3} \pi^{2} \frac{\mu_{\nu}^{2} \alpha^{2}}{m_{e}^{2} \omega_{0}} n_{e}
$$

Here the lines over $\sigma_{m}$ are to denote the cross sections per one target electron with coherence effects taken into account, and we have assumed $\omega_{0} \ll \omega$ in eq. (6.3).

The simplified approach we have adopted to evaluate the coherence effects, namely, to introduce the cutoff $p_{0} \sim 2 \pi / L_{0}$ on the electron recoil momentum, replace the structure factors $F\left(\vec{k}-\vec{k}^{\prime}-\vec{k}_{\gamma}\right)=F\left(\vec{p}^{\prime}\right)$ within the coherence volume $L_{0}^{3}$ by unity and then multiply the obtained elementary cross sections by the number electrons in the coherent volume, actually proves to be rather accurate. As we shall see, it just slightly overestimates the numerical factors in the cross sections (6.2) and (6.3). A more accurate estimate is obtained if one notes that for a macroscopically large number of electrons of the target contributing coherently to the cross section of the process, the summation in the expression for the structure factor in eq. (1.12) can be replaced by integration. This yields

$$
F\left(\vec{k}-\vec{k}^{\prime}-\vec{k}_{\gamma}\right) \simeq \frac{N_{e}}{V}(2 \pi)^{3} \delta^{3}\left(\vec{k}-\vec{k}^{\prime}-\vec{k}_{\gamma}\right),
$$

where $N_{e}=n_{e} V$ is the total electron number in the target and $V$ is the target's volume. Mutiplying the squared matrix element of the elementary process by

$$
\left|F\left(\vec{k}-\vec{k}^{\prime}-\vec{k}_{\gamma}\right)\right|^{2} \simeq N_{e} n_{e}(2 \pi)^{3} \delta^{3}\left(\vec{k}-\vec{k}^{\prime}-\vec{k}_{\gamma}\right)
$$

performing the integration over the momenta of the scattered neutrino and the recoil electron as well as over the directions of the photon emission and dividing by $N_{e}$, for the differential cross section per one target electron we obtain

$$
\frac{d \bar{\sigma}_{m}}{d \omega_{\gamma}}=\frac{\mu_{\nu}^{2} e^{4}}{4 \pi} \frac{\left(\omega-\omega_{\gamma}\right)}{\omega \omega_{\gamma}^{2}} \frac{n_{e}}{m_{e}^{2}},
$$

which has the same structure as (6.2), but is smaller by a factor of $3 / 2 \pi \simeq 0.5$. The $\omega_{\gamma}$-integrated cross section in the limit $\omega_{0} \ll \omega$ will be smaller than the expression in eq. (6.3) by the same factor. Note that the same approach applied to the radiative neutrinoelectron scattering mediated by the $\mathrm{NC}$ and $\mathrm{CC}$ weak interactions would yield vanishing cross sections of coherent scattering per target electron. This corresponds to the already discussed fact that in this case macroscopic coherence, though possible for sufficiently small electron recoils, would lead to vanishingly small cross sections.

Can the coherent enhancement of the neutrino magnetic moment mediated radiative neutrino scattering help us to increase the experimental sensitivity to the neutrino magnetic dipole moments or even to detect them? The best laboratory limits on neutrino magnetic moments come from the experiments on elastic $\nu e$ scattering at reactors, where one looks for possible deviations of the measured differential cross section from the usual one mediated by the weak $\mathrm{CC}$ and $\mathrm{NC}$ processes. The cross section due to the $\mu_{\nu}$-induced elastic $\nu e$ scattering is

$$
\frac{d \sigma_{m}^{e l}}{d T}=\frac{\mu_{\nu}^{2} e^{2}}{4 \pi}\left(\frac{1}{T}-\frac{1}{\omega}\right) \simeq \frac{\mu_{\nu}^{2} \alpha}{T}
$$


where $T$ is the kinetic energy of the recoil electron and in the last (approximate) equality it is assumed that $T \ll \omega$. This can be compared with the differential cross section (6.6) of the radiative neutrino-electron scattering, where a forward photon rather than the recoil electron is detected. For a numerical estimate, we set in eq. (6.6)

$$
n_{e}=N_{A} \rho\left(\mathrm{g} / \mathrm{cm}^{3}\right) \mathrm{Y}_{\mathrm{e}} \mathrm{cm}^{-3} \simeq(1.33 \mathrm{keV})^{3} \rho\left(\mathrm{g} / \mathrm{cm}^{3}\right),
$$

where $N_{A}$ is the Avogadro constant, $\rho$ is the density of the target material, $Y_{e}$ is the number of electrons per nucleon in the target, and in the last (approximate) equality we have set $Y_{e}=1 / 2$. In the regime $\omega_{\gamma} \ll \omega$ this gives

$$
\frac{d \bar{\sigma}_{m}}{d \omega_{\gamma}} \simeq 4 \pi \alpha^{2} \mu_{\nu}^{2} \frac{(1.33 \mathrm{keV})^{3}}{m_{e}^{2} \omega_{\gamma}^{2}} \rho\left(\mathrm{g} / \mathrm{cm}^{3}\right)
$$

Taking for an estimate $\omega_{\gamma} \sim 10 \mathrm{eV}$, which is about the smallest value that would allow to avoid the $\omega^{4}$ suppression of the radiative cross section, $\rho \sim 1 \mathrm{~g} / \mathrm{cm}^{3}$, and comparing eqs. (6.7) and (6.9), we find that even in the most optimistic case the cross section of coherently enhanced radiative neutrino-electron scattering exceeds that of the incoherent elastic scattering only for the electron recoil energies satisfying $T \gtrsim 100 \mathrm{keV}$. At the same time, reactor experiments are currently probing $\nu e$ scattering in the sub-keV region of the recoil energies $T$, where the cross section of the incoherent elastic scattering dominates. Still, it should be noted that experimentally detecting $\sim 10-100 \mathrm{eV}$ photons may be easier than detecting electron recoil energies in the same range.

A potentially important advantage of the coherent radiative $\mu_{\nu}$-mediated $\nu e$ scattering is that it could in principle allow detection of very low energy neutrinos. Consider, e.g., neutrinos of energy $\omega \sim 100 \mathrm{eV}$. For the elastic $\nu e$ scattering the electron recoil energies would then be $T \leq 2 \omega^{2} / m_{e} \simeq 0.04 \mathrm{eV}$, which is far too small to be measured in a foreseeable future. At the same time, detection of photons of energy $\sim 100 \mathrm{eV}$ which would be produced through coherent radiative $\nu e$ scattering does not pose any problem. However, the observability of the radiative process would depend crucially on the currently unknown values of the neutrino magnetic dipole moments (which, of course, applies to the elastic process as well). To give an idea of the magnitude of the expected cross section we rewrite below the expression for $d \bar{\sigma}_{m} / d \omega_{\gamma}$ given in eq. (6.9) in convenient units:

$$
\frac{d \bar{\sigma}_{m}}{d \omega_{\gamma}} \simeq 2.06 \times 10^{-56}\left(\frac{\mu_{\nu}}{10^{-12} \mu_{B}}\right)^{2} \rho\left(\mathrm{g} / \mathrm{cm}^{3}\right)\left(\frac{100 \mathrm{eV}}{\omega_{\gamma}}\right)^{2} \mathrm{~cm}^{2} / \mathrm{eV} .
$$

Here $\mu_{B}=e / 2 m_{e}$ is the electron Bohr magneton.

Turning now to radiative coherent axion-photon conversion on electrons considered in section 4 , we note that this process is similar to the neutrino magnetic moment induced radiative $\nu e$ scattering in that the interaction with electrons is mediated by the photon exchange and the constrained elementary cross section of the process scales as $p_{0}^{3}$ at small $p_{0}$. The assessment of the effects of possible macroscopic coherence is therefore also similar to the one for $\mu_{\nu}$-mediated $\nu e$ scattering. Following the procedure outlined at the beginning 
of this section, for the cross section per target electron with coherence effects taken into account we find

$$
\bar{\sigma}_{a} \simeq \frac{2}{3} \pi^{2} \frac{g_{a \gamma \gamma}^{2} \alpha^{2}}{m_{e}^{2} \omega} n_{e} \simeq \frac{2}{3} g_{a \gamma \gamma}^{2} \alpha^{2} \pi^{2} \frac{(1.33 \mathrm{keV})^{3}}{m_{e}^{2} \omega} \rho\left(\mathrm{g} / \mathrm{cm}^{3}\right)
$$

If instead one calculates the structure factor by replacing the summation by integration as in eq. (6.4), the result will differ from (6.11) by a factor of $3 / 2 \pi$.

It is instructive to compare this with other processes for axion detection. The most relevant process for experimental searches for relativistic axions is axion-photon conversion in an external magnetic field which is used in searches for axions from the sun with helioscopes. An axion traveling through a transverse magnetic field $B$ over a length $L$ is converted to photons with a probability $P$ given by [43]

$$
P=2.4 \times 10^{-21}\left(g_{a \gamma \gamma} \times 10^{10} \mathrm{GeV}\right)^{2}\left(\frac{B}{\mathrm{~T}}\right)^{2}\left(\frac{L}{\mathrm{~m}}\right)^{2} F,
$$

where the form factor

$$
F=\left(\frac{2 \sin \left(\frac{q L}{2}\right)}{q L}\right)^{2}
$$

accounts for the loss of coherence as a function of the momentum transfer $q$. For practical purposes $F \approx 1$ is a good approximation for the energy transfers of interest here. The photon production rate is $\Gamma_{a}=j_{a} \cdot P \cdot A_{\text {eff }}$, where $j_{a}$ is the flux of axions from a given source and $A_{\text {eff }}$ is the effective area of the detector. Realistic values for recent axion helioscopes such as CAST [44] are $B \approx 10 \mathrm{~T}, L \approx 10 \mathrm{~m}$ and $A_{\text {eff }} \approx 1 \mathrm{~cm}^{2}$ and we expect

$$
\Gamma_{a} \approx 2.4 \times 10^{-17} \mathrm{~cm}^{2}\left(g_{a \gamma \gamma} \times 10^{10} \mathrm{GeV}\right)^{2} j_{a} .
$$

The photon production rate due to the coherent radiative axion-photon conversion mechanism considered in section 4 is

$$
\Gamma_{a}=j_{a} \bar{\sigma}_{a} n_{e} V \simeq \bar{\sigma}_{a}(1.33 \mathrm{keV})^{3} \rho\left(\mathrm{g} / \mathrm{cm}^{3}\right) V j_{a},
$$

where $V$ denotes the volume of the detector, and the cross section per target electron $\bar{\sigma}_{a}$ was given in eq. (6.11). We can now compare the photon flux due to conversion in a magnetic field with the flux from radiative scattering on electrons. Taking for an estimate $\omega \simeq 3 \mathrm{keV}$, the characteristic energy of axions produced in the sun [45], and $\rho \simeq 1 \mathrm{~g} / \mathrm{cm}^{3}$, we find that the rate in eq. (6.15) is by far small compared to the magnetic conversion rate (6.14) for all reasonable detector volumes. Therefore, macroscopic coherence of the radiative axion conversion on electrons is not competitive with the coherent conversion in a magnetic field.

\section{Summary and discussion}

We have considered the possibility of achieving macroscopic coherence in neutrino detection experiments. For the elastic neutrino scattering processes, coherence at macroscopic 
scales can only be attained at the expense of unmeasurably small recoil energies of the target particles, $E_{\mathrm{rec}} \sim 10^{-43} \mathrm{eV}$, and so is of no use for neutrino detection. We therefore concentrated on radiative neutrino scattering on electrons $\nu e \rightarrow \nu e \gamma$, which has a number of attractive features:

- Unlike the elastic neutrino-nucleus scattering, elastic and radiative $\nu e$ scattering processes are sensitive to neutrino flavour and so could serve for studying neutrino flavour oscillations.

- In the case of radiative scattering the emitted photon rather than the recoil electron can be detected. As the photon energy $\omega_{\gamma}$ can be as large as the momentum $|\vec{q}|$ transferred to the electron from the neutrino, the process is sensitive to the neutrino momentum transfer rather than to the (very small) recoil energy of the target electron.

- An important advantage of the radiative $\nu e$ scattering is that neither the momentum transfer $|\vec{q}|$ nor the photon energy $\omega_{\gamma}$ need to be small in order to ensure macroscopic coherence of the process. What actually has to be small is the net recoil momentum of the target electron, which is the difference between the momentum transfer from the neutrinos $\vec{q}=\vec{k}-\vec{k}^{\prime}$ and the momentum $\vec{k}_{\gamma}$ carried away by the photon. This can happen even when both $|\vec{q}|$ and $\left|\vec{k}_{\gamma}\right|=\omega_{\gamma}$ are large compared to the inverse linear size of the target (or of a macroscopic volume within the target).

The drawback is that the requirement $\vec{k}-\vec{k}^{\prime} \simeq \vec{k}_{\gamma}$ puts a stringent constraint on the kinematics of the process, reducing the phase space accessible to the final-state particles and in general also affecting the dynamics of the process. This leads to a suppression of the cross section of the elementary process of neutrino radiative scattering on a single target electron. We have found that for the usual NC and CC induced $\nu e$ interactions, macroscopic enhancement of the number of electrons contributing coherently to the total cross section for small electron recoil momenta cannot overcome the suppression of the elementary cross section, and the net effect is a strong reduction of the total cross section compared to the incoherent case.

The situation is different for the radiative $\nu e$ scattering induced by neutrino magnetic (or electric) dipole moments. In that case the amplitude of the process is dynamically enhanced for $\vec{k}-\overrightarrow{k^{\prime}} \simeq \vec{k}_{\gamma}$ because of the propagator of the virtual photon being close to its mass-shell pole. The suppression of the elementary cross section due to the decrease of the phase space volume at small electron recoil momenta is then compensated by the macroscopically large number of electrons contributing coherently to the photon production rate. However, the cross section of the radiative process has some additional small factors (such as an extra power of $\alpha$ ) compared to the non-radiative one. At the same time, the usual increase of the radiative cross section at small photon energies is limited by the requirement that $\omega_{\gamma}$ exceed the characteristic atomic frequencies $\omega_{\text {at }}$ for neutrino scattering on atomic electrons or plasma frequency $\omega_{p}$ for scattering on free electrons in a conductor. As a consequence, even in the most optimistic case ( $\omega_{\gamma}$ close to its lower limit) for coherently enhanced radiative neutrino scattering the cross section per target electron exceeds the usual differential cross section of $\mu_{\nu}$-mediated $\nu e$ scattering only when in the 
latter case the kinetic energies of recoil electrons satisfies $T \gtrsim 100 \mathrm{keV}$. In any case, no increase of the experimental detection rates by a huge factor, which could be expected for a macroscopically coherent process, takes place.

The $\mu_{\nu}$-mediated coherent radiative $\nu e$ scattering has another advantage, though: it allows in principle to detect neutrinos in the energy domain $\sim 100 \mathrm{eV}-\mathrm{a}$ few $\mathrm{keV}$, which is currently not accessible to the experiment. Possible sources of such neutrinos include nuclear reactors, the sun and relic supernovae, whose neutrino spectrum can be softened by large redshifts. At the moment, the corresponding expected fluxes are essentially unknown, except for solar neutrinos, for which only one calculation in the keV energy range exists [41]. To detect keV-range neutrinos through the usual elastic $\mu_{\nu}$-mediated $\nu$ e scattering, one would need to measure the electron recoil energies on the order of $\sim 1 \mathrm{eV}$, whereas the current sensitivity is at the level of $\sim 0.3 \mathrm{keV}$. At the same time, detecting a $100 \mathrm{eV}-\mathrm{a}$ few keV photon does not pose any experimental problem. Obviously, whether or not such a detection of very low energy neutrinos will ever become possible depends crucially on the (currently unknown) values of the neutrino magnetic or electric dipole moments. This applies, of course, to both the radiative scattering discussed here and the usual elastic $\nu e$ scattering.

To summarize very briefly our findings, the elastic and radiative neutrino scattering processes that we have considered do not allow strong increase of neutrino detection cross sections through macroscopic coherence. For elastic scattering, the cross section per target particle can be increased by a huge factor $\sim N^{1 / 3}$, where $N$ is the total number of scatterers in the target; however, in this case macroscopic coherence requires neutrino scattering in practically forward direction with essentially zero momentum transfer and so with no observable signatures.

In the case of coherent radiative scattering, the emitted photons can in principle be easily detected, giving a clear experimental signature; however, the constraints on the kinematics of the process coming from the requirement of macroscopic coherence lead to very small cross sections per target particle. The only exception may be radiative scattering mediated by neutrino magnetic or electric dipole moments, but the experimental prospects for such processes are unclear because of the the unknown neutrino electromagnetic moments.

Does all this mean that macroscopically coherent detection of neutrinos is not possible in principle? We did not prove this as a theorem, but we believe that with our studies the observability of such a coherent enhancement becomes increasingly less probable.

We have also considered radiative axion photon conversion on electrons. Similarly to the case of $\mu_{\nu}$-mediated coherent radiative $\nu e$ scattering, the amplitude receives a dynamical enhancement in the kinematic regime of interest to us since the photon propagator goes almost on shell. Therefore, the decrease of the phase space volume is again to some extent compensated by the contribution of a macroscopic number of electrons. However, in this case the radiative conversion process should be compared with the most relevant detection process employed by ongoing experiments, i.e. the axion-photon conversion in an external magnetic field (the inverse Primakoff effect). This process is already macroscopically coherent, and it turns out that, despite the coherent enhancement, the radiative axion photon conversion studied in this paper is not competitive in practice. 
Can macroscopically coherent radiative scattering processes be employed for detecting dark matter particles? We have shown that for the conventionally discussed non-relativistic dark matter particle candidates this is not possible. The coherent enhancement mechanism studied here may, however, work for the detection of relativistic particles which usually exist in multi-component dark matter models, an example being the "boosted" dark matter [42]. We believe that such a possibility deserves a dedicated study.

\section{Acknowledgments}

The authors are grateful to Werner Maneschg and Alexei Smirnov for useful discussions.

\section{A Kinematics of the process}

Consider the kinematics of the process

$$
X(k)+e(p) \rightarrow X\left(k^{\prime}\right)+e\left(p^{\prime}\right)+\gamma\left(k_{\gamma}\right),
$$

where $X$ is a projectile particle of mass $M$. In the rest frame of the initial-state electron the 4-momenta of the incident $X$-particle, initial-state electron, scattered $X$-particle, finalstate electron and emitted photon are, respectively,

$$
k=(\omega, \vec{k}), \quad p=\left(m_{e}, \overrightarrow{0}\right), \quad k^{\prime}=\left(\omega^{\prime}, \vec{k}^{\prime}\right), \quad p^{\prime}=\left(E_{p^{\prime}}, \vec{p}^{\prime}\right), \quad k_{\gamma}=\left(\omega_{\gamma}, \vec{k}_{\gamma}\right),
$$

where

$$
\omega=\sqrt{\vec{k}^{2}+M^{2}}, \quad \omega^{\prime}=\sqrt{\vec{k}^{2}+M^{2}}, \quad E_{p^{\prime}}=\sqrt{\vec{p}^{\prime 2}+m_{e}^{2}}, \quad \omega_{\gamma}=\left|\vec{k}_{\gamma}\right| .
$$

The energy and momentum conservation laws yield

$$
\begin{aligned}
& \omega=\omega^{\prime}+\left(E_{p^{\prime}}-m_{e}\right)+\omega_{\gamma}, \\
& \vec{k}=\vec{k}^{\prime}+\vec{p}^{\prime}+\vec{k}_{\gamma} .
\end{aligned}
$$

In what follows we will be assuming the recoil electron to be non-relativistic and will neglect its kinetic energy. The energy conservation condition then simplifies to

$$
\omega=\omega^{\prime}+\omega_{\gamma} .
$$

Expressing $\vec{k}^{\prime}$ from eq. (A.5) and substituting it into (A.6), we get

$$
\left(\vec{k}-\vec{k}_{\gamma}-\vec{p}^{\prime}\right)^{2}=\vec{k}^{2}-2 \omega_{\gamma} \sqrt{\vec{k}^{2}+M^{2}}+\omega_{\gamma}^{2},
$$

or

$$
\vec{p}^{\prime 2}-2\left|\vec{p}^{\prime}\right| R \cos \theta_{\vec{p}^{\prime}\left(\vec{k}-\vec{k}^{\prime}\right)}-2|\vec{k}| \omega_{\gamma} \cos \theta_{\gamma}=-2 \omega_{\gamma} \sqrt{\vec{k}^{2}+M^{2}} .
$$

Here $\theta_{\gamma}$ is the angle between $\vec{k}_{\gamma}$ and $\vec{k}, \theta_{\vec{p}^{\prime}\left(\vec{k}-\vec{k}^{\prime}\right)}$ is the angle between $\vec{p}^{\prime}$ and $\vec{k}-\vec{k}_{\gamma}$, and

$$
R \equiv\left|\vec{k}-\vec{k}_{\gamma}\right|=\sqrt{\vec{k}^{2}+\omega_{\gamma}^{2}-2 \omega_{\gamma}|\vec{k}| \cos \theta_{\gamma}}
$$


We will be interested in the regime of very small $\left|\vec{p}^{\prime}\right|$. Let us first demonstrate that for $M \neq 0$ the quantity $\left|\vec{p}^{\prime}\right|$ cannot be arbitrarily small. Indeed, in the limit $\vec{p}^{\prime} \rightarrow 0$ eq. (A.8) leads to unphysical $\cos \theta_{\gamma}=\sqrt{\vec{k}^{2}+M^{2}} /|\vec{k}|>1$. Next, we assume $\left|\vec{p}^{\prime}\right|$ to be non-zero but small, such that the $\vec{p}^{\prime 2}$ term in (A.8) can be neglected. We should then also replace $\cos \theta_{\gamma}$ in the factor $R$ by unity, i.e. set $R=|| \vec{k}\left|-\omega_{\gamma}\right|$. This is because $R$ enters in eq. (A.8) multiplied by $\left|\vec{p}^{\prime}\right|$, and for small $\left|\vec{p}^{\prime}\right|$ the difference $1-\cos \theta_{\gamma}=\mathcal{O}\left(\left|\vec{p}^{\prime}\right|\right)$; thus, keeping $1-\cos \theta_{\gamma}$ in $R$ would lead to terms of higher order of smallness in $\left|\vec{p}^{\prime}\right|$. Requiring $\cos \theta_{\gamma} \leq 1$, we then find from eq. (A.8)

$$
\sqrt{\vec{k}^{2}+M^{2}}-|\vec{k}| \leq \frac{|| \vec{k}\left|-\omega_{\gamma}\right|}{\omega_{\gamma}} \cdot\left|\vec{p}^{\prime}\right| \cos \theta_{\vec{p}^{\prime}\left(\vec{k}-\vec{k}^{\prime}\right)}
$$

For non-relativistic projectile particles $(|\vec{k}| \ll M)$, eq. (A.10) yields

$$
M-\frac{|\vec{k}|}{\omega_{\gamma}} \cdot\left|\vec{p}^{\prime}\right| \cos \theta_{\vec{p}^{\prime}\left(\vec{k}-\vec{k}^{\prime}\right)} \leq|\vec{k}|
$$

which means that the two terms on the left hand side should nearly cancel each other. This requires

$$
\omega_{\gamma} \simeq \frac{|\vec{k}|}{M}\left|\vec{p}^{\prime}\right| \cos \theta_{\vec{p}^{\prime}\left(\vec{k}-\vec{k}^{\prime}\right)} \ll\left|\vec{p}^{\prime}\right| .
$$

As discussed in section 1.1, to achieve macroscopic coherence one needs $\left|\vec{p}^{\prime}\right| \lesssim 10^{-5} \mathrm{eV}$; condition (A.12) then implies that the requirement $\omega_{\gamma} \gtrsim \omega_{\text {at }}$ (or $\omega_{\gamma} \gtrsim \omega_{p}$ for scattering on free electrons in a conductor) is badly violated for non-relativistic projectiles, leading to a strong suppression of the cross section of process (A.1). Similar estimates apply and the same conclusion holds for the case of moderately relativistic projectiles. ${ }^{19}$

In the ultra-relativistic regime $|\vec{k}| \gg M$ eq. (A.10) yields an upper bound on $M$ :

$$
M^{2} \leq 2 \omega \frac{\omega-\omega_{\gamma}}{\omega_{\gamma}}\left|\vec{p}^{\prime}\right| \cos \theta_{\vec{p}^{\prime}\left(\vec{k}-\vec{k}^{\prime}\right)}
$$

For $\omega_{\gamma}$ not too close to its upper limit $\omega$ and $\cos \theta_{\vec{p}^{\prime}\left(\vec{k}-\vec{k}^{\prime}\right)} \sim 1$ eq. (A.13) yields $M^{2} \lesssim 2 \omega\left|\vec{p}^{\prime}\right|$. For $\left|\vec{p}^{\prime}\right| \sim 10^{-5} \mathrm{eV}$ and $\omega \sim 1 \mathrm{keV}$ this gives $M \lesssim 0.14 \mathrm{eV}$, which can be readily satisfied when the projectiles are neutrinos or axions.

\section{B 3-body phase space volume}

We shall now consider the regime of relativistic projectiles assuming that condition (A.13) is satisfied with a large margin. This will allow us to treat the projectile as essentially massless.

Consider the 3-body phase space volume integral

$$
R_{3} \equiv \int \frac{d^{3} p^{\prime}}{2 E_{p^{\prime}}} \frac{d^{3} k_{\gamma}}{2 \omega_{\gamma}} \frac{d^{3} k^{\prime}}{2 \omega^{\prime}} \delta^{3}\left(\vec{k}-\vec{k}^{\prime}-\vec{p}^{\prime}-\vec{k}_{\gamma}\right) \delta\left(\omega-\omega^{\prime}-\omega_{\gamma}\right)
$$

\footnotetext{
${ }^{19}$ It is convenient to write in this case $\sqrt{\vec{k}^{2}+M^{2}}=|\vec{k}|(1+a)$, where $a=\mathcal{O}(1)$. Noting that the factor $\left[|\vec{k}|-\omega_{\gamma}\right] / \omega_{\gamma}$ is maximised for $\omega_{\gamma} \ll|\vec{k}|$, we find from (A.10) $a \omega_{\gamma} \leq\left|\vec{p}^{\prime}\right| \cos \theta_{\vec{p}^{\prime}\left(\vec{k}-\vec{k}^{\prime}\right)} \ll \omega_{\text {at }}$.
} 
in the limit of non-relativistic energies of the recoil electrons. We will calculate it in two cases: (i) without additionally constraining $\left|\vec{p}^{\prime}\right|$ and (ii) assuming that $\left|\vec{p}^{\prime}\right|$ is limited from above by a small value $p_{0}$. A similar approach is used in the computations of the cross sections given in section 2 .

We start by finding $R_{3}$ without additionally constraining the electron recoil momentum $\vec{p}^{\prime}$. It is convenient to first integrate over $\vec{p}^{\prime}$ by making use of the $\delta^{3}$-function enforcing 3-momentum conservation. A straightforward calculation then yields

$$
R_{3}=\frac{\pi^{2} \omega^{3}}{3 m_{e}} .
$$

Next, we will calculate $R_{3}$ using a different integration order, which will be more convenient for studying the case of constrained $\left|\vec{p}^{\prime}\right|$. To this end, we use the $\delta^{3}$-function to integrate over the momentum $\vec{k}^{\prime}$ of the scattered projectile. One then has to substitute

$$
\vec{k}^{\prime}=\vec{k}-\vec{p}^{\prime}-\vec{k}_{\gamma}
$$

in the integrand of the remaining integral. Using $\delta\left(\omega-\omega^{\prime}-\omega_{\gamma}\right)=2 \omega^{\prime} \delta\left[\left(\omega-\omega_{\gamma}\right)^{2}-\omega^{\prime 2}\right]$ and $\omega^{\prime 2}=\vec{k}^{\prime 2}=\left(\vec{k}-\vec{p}^{\prime}-\vec{k}_{\gamma}\right)^{2}$, we find

$$
\delta\left[\left(\omega-\omega_{\gamma}\right)^{2}-\omega^{\prime 2}\right]=\delta\left(2\left|\vec{p}^{\prime}\right| R \cos \theta_{\vec{p}^{\prime}\left(\vec{k}-\vec{k}_{\gamma}\right)}-\vec{p}^{\prime 2}-2 \omega \omega_{\gamma}\left(1-\cos \theta_{\gamma}\right)\right),
$$

where $\theta_{\gamma}$ is the angle between $\vec{k}_{\gamma}$ and $\vec{k}$ and

$$
R \equiv\left|\vec{k}-\vec{k}_{\gamma}\right|=\sqrt{\left(\omega-\omega_{\gamma}\right)^{2}+2 \omega \omega_{\gamma} x_{\gamma}}, \quad x_{\gamma} \equiv 1-\cos \theta_{\gamma} .
$$

Requiring $\cos \theta_{\vec{p}^{\prime}\left(\vec{k}-\vec{k}_{\gamma}\right)} \leq 1$, we find that for fixed $\omega$ and $x_{\gamma}$ the quantity $\left|\vec{p}^{\prime}\right|$ must lie in the interval $\left[p_{\min }^{\prime}, p_{\max }^{\prime}\right]$, where

$$
p_{\min }^{\prime}=R-\left(\omega-\omega_{\gamma}\right), \quad p_{\max }^{\prime}=R+\left(\omega-\omega_{\gamma}\right) .
$$

We shall now consider the case when $\left|\vec{p}^{\prime}\right|$ is limited from above by a value $p_{0}<p_{\max }^{\prime}$. The integration over $\left|\vec{p}^{\prime}\right|$ is then done in the interval $\left|\vec{p}^{\prime}\right| \in\left[p_{\text {min }}^{\prime}, p_{0}\right]$. From the condition $p_{0}>p_{\text {min }^{\prime}}$ we find that $\cos \theta_{\gamma}$ must be in the interval given in eq. (2.13). Small $p_{0}$ therefore means that the photon is emitted in a nearly forward direction with respect to the incident projectile. From the energy-momentum conservation relations (A.5) and (A.4) and the fact that we consider ultra-relativistic projectiles it follows that the same is true for the scattered projectile particle, i.e. for small $p_{0}$ its momentum $\vec{k}^{\prime}$ is also nearly parallel to $\vec{k}$.

Note that in general $0 \leq 1-\cos \theta_{\gamma} \leq 2$. Therefore, the condition $\left|\vec{p}^{\prime}\right| \leq p_{0}$ puts a non-trivial constraint on $x_{\gamma}=1-\cos \theta_{\gamma}$ only when the expression on the right hand side of eq. (2.13) is smaller than 2. This yields $p_{0} / 2<\omega_{\gamma}$. On the other hand, the constraint $\left|\vec{p}^{\prime}\right| \leq p_{0}$ is only non-trivial when $p_{0}<p_{\max }^{\prime}$ for all $x_{\gamma}$. Setting $x_{\gamma}=0$ (which minimizes $p_{\max }^{\prime}$ for a given $\omega_{\gamma}$ ) then yields $\omega_{\gamma} \leq \omega-p_{0} / 2$. Thus, in the constrained case under consideration the allowed range for the photon energy is

$$
p_{0} / 2 \leq \omega_{\gamma} \leq \omega-p_{0} / 2
$$


Since we are interested in tiny values of $p_{0}$, for all practical purposes the interval (B.7) can be replaced by the usual allowed range for the photon energy, $0 \leq \omega_{\gamma} \leq \omega$.

Performing in (B.1) the integration over $\vec{k}^{\prime}$ by making use of the $\delta^{3}$ function, then integrating over the directions of the vector $\vec{p}^{\prime}$ with the help of (B.4), over the modulus of this vector in the interval $\left[p_{\min }, p_{0}\right]$ and finally over $\vec{k}_{\gamma}$ (taking eq. (B.6) into account), we find

$$
R_{3}=\frac{\pi^{2} p_{0}^{3}}{6 m_{e}} .
$$

This has to be compared with the unconstrained result (B.2).

\section{Squared matrix elements}

In this appendix we collect the expressions for the squared moduli of the transition matrix elements $\overline{|\mathcal{M}|^{2}}$ for the processes considered in sections 2 and 4 . Here, as usual, the line over $|\mathcal{M}|^{2}$ denotes the summation over the polarisations of the final particles and averaging over the polarisations of the initial-state ones.

For weak NC and CC induced radiative neutrino scattering, the calculations are most easily done in the Coulomb gauge. From eq. (2.3) one finds

$$
\begin{aligned}
\overline{\left|\mathcal{M}_{w}\right|^{2}}= & \frac{G_{F}^{2} g_{V}^{2} e^{2}}{2} 32\left\{\frac{1}{\omega_{\gamma}^{2}}\left[\vec{p}^{\prime 2}-\frac{\left(\vec{p}^{\prime} \vec{k}_{\gamma}\right)^{2}}{\omega_{\gamma}^{2}}\right]\left(\omega \omega^{\prime}+\vec{k} \vec{k}^{\prime}\right)+2\left(\omega \omega^{\prime}-\frac{\left(\vec{k} \vec{k}_{\gamma}\right)\left(\vec{k}^{\prime} \vec{k}_{\gamma}\right)}{\omega_{\gamma}^{2}}\right)\right. \\
& \left.-\frac{2}{\omega_{\gamma}}\left[\omega\left(\vec{p}^{\prime} \vec{k}^{\prime}-\frac{\left(\vec{p}^{\prime} \vec{k}_{\gamma}\right)\left(\vec{k}^{\prime} \vec{k}_{\gamma}\right)}{\omega_{\gamma}^{2}}\right)+\omega^{\prime}\left(\vec{p}^{\prime} \vec{k}-\frac{\left(\vec{p}^{\prime} \vec{k}_{\gamma}\right)\left(\vec{k} \vec{k}_{\gamma}\right)}{\omega_{\gamma}^{2}}\right)\right]\right\} .
\end{aligned}
$$

For neutrino magnetic (or electric) dipole moment induced radiative scattering, Lorentz gauge proves to be more convenient because it allows one to more easily get rid of angledependent denominators in most terms and thus to simplify the subsequent angular integrations. From eq. (2.16) we obtain

$$
\begin{aligned}
\overline{\left|\mathcal{M}_{m}\right|^{2}}= & \frac{\mu_{\nu}^{2} e^{4}}{\left(-2 k k^{\prime}\right)^{2}} 8\left(k k^{\prime}\right) \\
& \times\left\{2\left[\frac{\left[p\left(k+k^{\prime}\right)\right]\left[p^{\prime}\left(k+k^{\prime}\right)\right]}{\left(p k_{\gamma}\right)\left(p^{\prime} k_{\gamma}\right)}-1\right]\left(k k^{\prime}\right)-m_{e}^{2}\left[\frac{p\left(k+k^{\prime}\right)}{p^{\prime} k_{\gamma}}-\frac{p^{\prime}\left(k+k^{\prime}\right)}{p k_{\gamma}}\right]^{2}\right\} .
\end{aligned}
$$

Calcualations for radiative axion-photon conversion are also more easily done in the Lorentz gauge. Since the complete expression for the squared matrix element is quite lengthy in that case (mostly due to the interference of the two parts of the amplitudes corresponding to the interchange of the 4-momenta of the two photons in the final state), we give here only the expression in the limit of small $\left|\vec{p}^{\prime}\right|$ that is of main interest to us:

$$
\overline{\left|\mathcal{M}_{a}\right|^{2}} \simeq 4 g_{a \gamma \gamma}^{2} e^{4}\left(\frac{\omega_{1}}{\omega_{2}}+\frac{\omega_{2}}{\omega_{1}}\right) .
$$

Open Access. This article is distributed under the terms of the Creative Commons Attribution License (CC-BY 4.0), which permits any use, distribution and reproduction in any medium, provided the original author(s) and source are credited. 


\section{References}

[1] COHERENT collaboration, D. Akimov et al., Observation of Coherent Elastic Neutrino-Nucleus Scattering, Science 357 (2017) 1123 [arXiv:1708.01294] [InSPIRE].

[2] COHERENT collaboration, D. Akimov et al., COHERENT Collaboration data release from the first observation of coherent elastic neutrino-nucleus scattering, arXiv:1804.09459 [INSPIRE].

[3] D.Z. Freedman, Coherent Neutrino Nucleus Scattering as a Probe of the Weak Neutral Current, Phys. Rev. D 9 (1974) 1389 [INSPIRE].

[4] D.Z. Freedman, D.N. Schramm and D.L. Tubbs, The Weak Neutral Current and Its Effects in Stellar Collapse, Ann. Rev. Nucl. Part. Sci. 27 (1977) 167 [InSPIRE].

[5] W. Maneschg, The Status of CONUS, talk given at the XXVIIIth International Conference on Neutrino Physics and Astrophysics "Neutrino 2018", Heidelberg Germany (2018), https://zenodo.org/record/1286927\#.WzCpdxx9hB8.

[6] Yu. V. Gaponov, Yu. L. Dobrynin and V.N. Tikhonov, Neutrino and anti-neutrino Scattering on Hydrogen-Like Atom, Yad. Fiz. 22 (1975) 328 [Yad. Fiz. 22 (1975) 328] [INSPIRE].

[7] Yu.V. Gaponov and V.N. Tikhonov, Elastic scattering of low-energy neutrinos by atomic systems, Sov. J. Nucl. Phys. 26 (1977) 314 [Yad. Fiz. 26 (1977) 594].

[8] L.M. Sehgal and M. Wanninger, Atomic Effects in Coherent Neutrino Scattering, Phys. Lett. B 171 (1986) 107 [INSPIRE].

[9] J. Weber, Gravitons, neutrinos, and anti-neutrinos, Found. Phys. 14 (1984) 1185 [INSPIRE].

[10] J. Weber, Method for observation of neutrinos and antineutrinos, Phys. Rev. C 31 (1985) 1468 [INSPIRE].

[11] J. Weber, Apparent observation of abnormally large coherent scattering cross-sections using keV and meV range anti-neutrinos, and solar neutrinos, Phys. Rev. D 38 (1988) 32 [INSPIRE].

[12] P.G. Debrunner and H. Frauenfelder, Introduction to the Mössbauer effect, in An introduction to Mössbauer spectroscopy, L. May eds., Plenum Press, New York U.S.A. (1971).

[13] G.F. Bertsch and S.M. Austin, Comment on 'Method for Observation of Neutrinos and Anti-neutrinos.', Phys. Rev. C 34 (1986) 361 [InSPIRE].

[14] T.H. Ho, Comments on the 'Method for observation of neutrinos and anti-neutrinos.', Phys. Lett. B 168 (1986) 295.

[15] R.C. Casella, On the theory of coherent neutrino scattering from crystals, Nuovo Cim. A 94 (1986) 42 [INSPIRE].

[16] M.N. Butler, Comment on 'Method for observation of neutrinos and anti-neutrinos.', Phys. Rev. C 35 (1987) 1164 [InSPIRE].

[17] Y. Aharonov, F.T. Avignone, A. Casher and S. Nussinov, Constraints on Anomalous Scattering of Neutrinos From Crystals, Phys. Rev. Lett. 58 (1987) 1173 [INSPIRE].

[18] P.F. Smith, Comments on a reported measurement of an abnormally large coherent neutrino cross-section, Phys. Lett. B 183 (1987) 107 [INSPIRE].

[19] H.J. Lipkin, The N Dependence of Coherent Radiation From Crystals, Phys. Rev. Lett. 58 (1987) 1176 [InSPIRE]. 
[20] G.T. Trammell and J.P. Hannon, Comment on " $N$ dependence of coherent radiation from crystals", Phys. Rev. Lett. 61 (1988) 653.

[21] J.D. Franson and B.C. Jacobs, Null result for enhanced neutrino scattering in crystals, Phys. Rev. A 46 (1992) 2235 [INSPIRE].

[22] M.P. McHugh and P.T. Keyser, Test of coherent neutrino detection using sapphire crystals, talk given at the 28th Rencontres de Moriond: Perspectives in Neutrinos, Atomic Physics and Gravitation, Villars sur Ollon Switzerland (1993).

[23] L.D. Landau and E.M. Lifshitz, Electrodynamics of Continuous Media, second edition revised and enlarged, Pergamon Press, Oxford U.K. (1984).

[24] C. Callan, F. Dyson and S. Treiman, Neutrino Detection Primer, Jason Report JSR-84-105 (1988), unpublished.

[25] T.D. Lee and A. Sirlin, Possible Method of Determining the Moment of Charge of $\nu_{e}$, Rev. Mod. Phys. 36 (1964) 666 [inSPIRE].

[26] E.D. Zhizhin, R.V. Konoplich and Y.P. Nikitin, Neutrino (anti-neutrinos) electron scattering with bremsstrahlung in the renormalizable theory of weak and electromagnetic interaction, Izv. Vuz. Fiz. 1975 (1975) 82.

[27] A.M. Mourao, L. Bento and B.K. Kerimov, Effects of the mass and magnetic moment of the neutrinos in $\nu e \rightarrow \nu e \gamma$, Phys. Lett. B 237 (1990) 469 [INSPIRE].

[28] A. Loeb and G.D. Starkman, A detector for the cosmic neutrino background, Institute of Advanced Studies preprint IASSNS-AST 90/10 (1990), unpublished.

[29] S. Bahcall and A. Gould, Can cosmic neutrinos be detected by Bremsstrahlung from a metal?, Phys. Rev. D 43 (1991) 940 [InSPIRE].

[30] A. Weber and L.M. Sehgal, CVC and PCAC in neutrino-lepton interactions, Nucl. Phys. B 359 (1991) 262 [InSPIRE].

[31] F. Buccella, C. Gualdi, G. Miele and P. Santorelli, Electron Bremsstrahlung induced by neutrinos with magnetic and electric dipole momenta, Nuovo Cim. B 107 (1992) 1343 [INSPIRE].

[32] J. Bernabeu, S.M. Bilenky, F.J. Botella and J. Segura, Neutrino magnetic moment and the process $\nu e \rightarrow \nu e \gamma$, Nucl. Phys. B 426 (1994) 434 [hep-ph/9404341] [InSPIRE].

[33] J.D. Jackson, Classical Electrodynamics, third edition, Wiley, New York U.S.A. (1999).

[34] J.P.J. Carney, R.H. Pratt, L. Kissel, S.C. Roy and S.K. Sen Gupta, Rayleigh scattering from excited states of atoms and ions, Phys. Rev. A 61 (2000) 052714.

[35] J. Fišák, J. Krtička, D. Munzar and J. Kubát, Rayleigh scattering in the atmospheres of hot stars, Astron. Atrophys. A 95 (2016) 590.

[36] C. Kouvaris and J. Pradler, Probing sub-GeV Dark Matter with conventional detectors, Phys. Rev. Lett. 118 (2017) 031803 [arXiv:1607.01789] [INSPIRE].

[37] G.G. Raffelt, Astrophysical axion bounds diminished by screening effects, Phys. Rev. D 33 (1986) 897 [INSPIRE].

[38] P. Sikivie, Experimental Tests of the Invisible Axion, Phys. Rev. Lett. 51 (1983) 1415 [Erratum ibid. 52 (1984) 695] [INSPIRE]. 
[39] P.M. Bergstrom, Jr., T. Surić, K. Pisk and R.H. Pratt, Compton scattering of photons from bound electrons: full relativistic independent-particle-approximation calculations, Phys. Rev. A 48 (1993) 1134.

[40] P.P. Kane, L. Kissel, R.H. Pratt and S.C. Roy, Elastic Scattering of $\gamma$ Rays and X-rays by Atoms, Phys. Rept. 140 (1986) 75 [inSPIRE].

[41] E. Vitagliano, J. Redondo and G. Raffelt, Solar neutrino flux at keV energies, JCAP 12 (2017) 010 [arXiv: 1708.02248] [INSPIRE].

[42] K. Agashe, Y. Cui, L. Necib and J. Thaler, (In)direct Detection of Boosted Dark Matter, JCAP 10 (2014) 062 [arXiv: 1405.7370] [INSPIRE].

[43] K. Van Bibber, N.R. Dagdeviren, S.E. Koonin, A. Kerman and H.N. Nelson, Proposed experiment to produce and detect light pseudoscalars, Phys. Rev. Lett. 59 (1987) 759 [INSPIRE].

[44] CAST collaboration, K. Zioutas et al., First results from the CERN Axion Solar Telescope (CAST), Phys. Rev. Lett. 94 (2005) 121301 [hep-ex/0411033] [INSPIRE].

[45] K. van Bibber, P.M. McIntyre, D.E. Morris and G.G. Raffelt, A Practical Laboratory Detector for Solar Axions, Phys. Rev. D 39 (1989) 2089 [inSPIRE]. 